\title{
Local lung hypoxia determines epithelial fate decisions during alveolar regeneration
}

\author{
Ying Xi ${ }^{1,6}$, Thomas Kim ${ }^{1}$, Alexis N. Brumwell ${ }^{1}$, Ian H. Driver ${ }^{2}$, Ying Wei ${ }^{1}$, Victor $\operatorname{Tan}^{1}$, Julia R. Jackson ${ }^{1}$, \\ Jianming $\mathrm{Xu}^{3}$, Dong-Kee Lee ${ }^{3}$, Jeffrey E. Gotts ${ }^{1}$, Michael A. Matthay ${ }^{1}$, John M. Shannon ${ }^{4}$, Harold A. Chapman ${ }^{1,7,8}$ \\ and Andrew E. Vaughan ${ }^{1,5,6,7}$
}

After influenza infection, lineage-negative epithelial progenitors (LNEPs) exhibit a binary response to reconstitute epithelial barriers: activating a Notch-dependent $\Delta \mathrm{Np} 63 /$ cytokeratin 5 (Krt5) remodelling program or differentiating into alveolar type II cells (AEC2s). Here we show that local lung hypoxia, through hypoxia-inducible factor (HIF1 $\alpha$ ), drives Notch signalling and Krt5pos basal-like cell expansion. Single-cell transcriptional profiling of human AEC2s from fibrotic lungs revealed a hypoxic subpopulation with activated Notch, suppressed surfactant protein C (SPC), and transdifferentiation toward a Krt5 $5^{\text {pos }}$ basal-like state. Activated murine Krt5 ${ }^{\text {pos }}$ LNEPs and diseased human AEC2s upregulate strikingly similar core pathways underlying migration and squamous metaplasia. While robust, HIF1 $\alpha$-driven metaplasia is ultimately inferior to AEC2 reconstitution in restoring normal lung function. HIF $1 \alpha$ deletion or enhanced Wnt/ $\beta$-catenin activity in Sox $2^{\text {pos }}$ LNEPs blocks Notch and Krt5 activation, instead promoting rapid AEC2 differentiation and migration and improving the quality of alveolar repair.

The adult lung is a largely quiescent tissue but responds effectively to injury by activating stem/progenitor populations and promoting proliferation of surviving, mature lineages. Depending on the type and severity of cellular injury, different cell types are involved in repair ${ }^{1-5}$. A remarkable expansion of potentially regenerative $\Delta \mathrm{Np} 3^{\mathrm{pos}} / \mathrm{Krt5}^{\text {pos }}$ cells was observed in mouse lungs infected by murine-adapted H1N1 (PR8) influenza A virus ${ }^{6}$. Lineage-tracing experiments showed that $>80 \%$ of the expanded $\mathrm{Krt}^{\mathrm{pos}}$ cells arise from LNEP populations in distal airways and probably alveoli ${ }^{2}$. These LNEPs proliferate and migrate dramatically to damaged sites to reconstitute the alveolar epithelium and promote recovery. Specific ablation of the newly expanded $\mathrm{Krt} 5^{\text {pos }}$ cells by diphtheria toxin resulted in prolonged hypoxemia, confirming involvement of these cells in the repair process ${ }^{7}$. Notch signalling is critical for activating this $\Delta \mathrm{Np} 63 / \mathrm{Krt} 5$ remodelling program; however, persistent Notch signalling prevents alveolar epithelial differentiation ${ }^{8}$, leading to cystic structures indicative of incomplete regeneration. Similar $\mathrm{Krt} 5^{\text {pos }}$ honeycombing cysts also exist in the lungs from patients with pulmonary fibrosis, suggesting that common mechanisms apply to injury responses in both mice and humans ${ }^{2}$.
In this study we explored the basis for activation and expansion of lung epithelial stem/progenitor cells observed in mice infected with H1N1 (PR8) influenza. Mice and humans infected with H1N1 influenza develop large areas virtually devoid of alveolar epithelial cells, requiring both expansion and migration of surviving AEC2s and more undifferentiated progenitors to restore alveolar epithelial barriers ${ }^{2,9,10}$. Studies here interrogate the underlying mechanisms and sources of epithelial progenitors that determine the alternative pathways of alveolar repair.

\section{RESULTS}

Influenza-activated $\mathrm{Krt5} 5^{\text {pos }}$ cells are derived from $\mathrm{p} 63^{\text {pos }}$ lineage-negative progenitors

Our previous studies indicated that LNEPs are a Sox $2^{\text {pos }}$ heterogeneous population comprised of both cells expressing the stem cell transcription factor $\Delta \mathrm{Np} 63$ and $\Delta \mathrm{Np} 63^{\text {neg }}$ cells, although p63 expression was restricted to a minor LNEP fraction ${ }^{2,11}$. Transplantation studies suggested the possibility that these subtypes are biased toward either basal-like or AEC2 differentiation, respectively ${ }^{2}$. To clarify this, we infected tamoxifen-treated

${ }^{1}$ Department of Medicine, Cardiovascular Research Institute, UCSF, San Francisco, California 94143, USA. ${ }^{2}$ Department of Anatomy, UCSF, San Francisco, California 94143, USA. ${ }^{3}$ Department of Molecular and Cellular Biology, Baylor College of Medicine, Houston, Texas 77030, USA. ${ }^{4}$ Division of Pulmonary Biology, Cincinnati Children's Hospital Medical Center, Cincinnati, Ohio 45229, USA. Institute for Regenerative Medicine, University of Pennsylvania, Philadelphia, Pennsylvania 19104, USA. ${ }^{6}$ Present addresses: Department of Immunology, Genentech Inc., South San Francisco, California 94080, USA (Y.X.); Department of Biomedical Sciences, School of Veterinary Medicine, University of Pennsylvania, Philadelphia, Pennsylvania 19104, USA (A.E.V.). ${ }^{7}$ These authors jointly supervised this work.

${ }^{8}$ Correspondence should be addressed to H.C. (e-mail: hal.chapman@ucsf.edu)

Received 17 March 2017; accepted 21 June 2017; published online 24 July 2017; DOI: 10.1038/ncb3580 
a

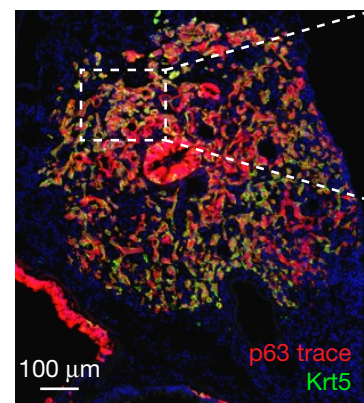

C

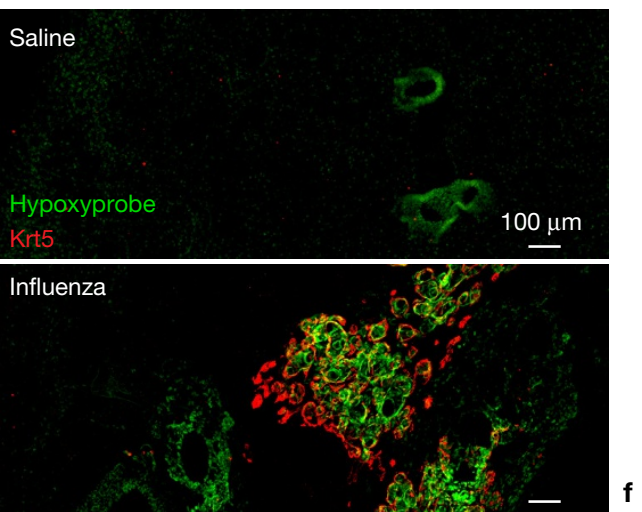

d
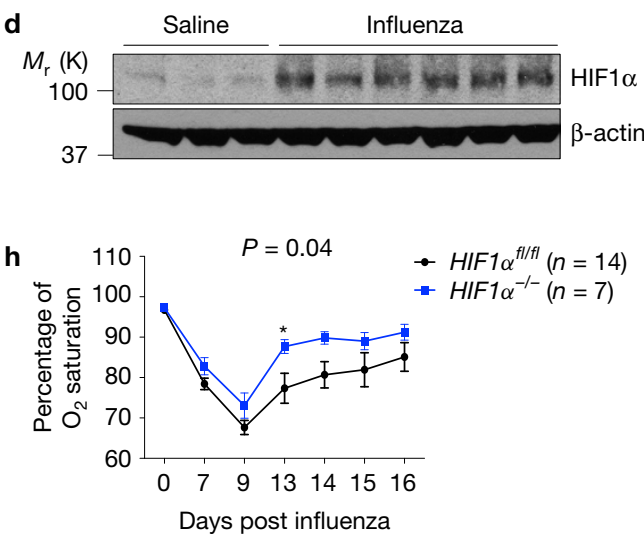

Figure 1 Epithelial HIF $1 \alpha$ deletion blocks alveolar Krt5 activation post H1N1 infection. (a) Krt5 $5^{\text {pos }}$ cells are completely traced (tdTomatopos) after influenza injury in p63-CreERT2/tdTomato mice. (b) Quantification of lineage tracing by manual cell counts in tissue sections. Data are mean \pm s.e.m., $n=3$ mice. (c) Krt5pos cells (red) invariably appear in hypoxic alveolar regions (hypoxyprobe, green) after influenza injury in mice, although some $\mathrm{Krt5}^{\text {neg }}$ hypoxic regions were also observed ( $n=7$ mice). (d) HIF $1 \alpha$ protein accumulates in influenza-infected mouse lungs ( $n=6$ influenza, $n=3$ saline, $\beta$-actin as loading control). (e) Alveolar Krt5 $5^{\text {pos }}$ expansion (green) (left) is largely blocked by epithelial HIFl $\alpha$ deletion (right). (f) Quantification of e. Data are mean \pm s.e.m., $n=5$ wild-type, $n=4 H I F l \alpha^{-/-}$mice from two independent experiments. (g) Krt5 mRNA levels are reduced in $H I F 1 \alpha^{-/-}$mice. Data are mean \pm s.e.m., $n=8$ wild-type, $n=9 \mathrm{HIFl} \alpha^{-/-}$ mice from 3 independent experiments. (h) Arterial oxygen saturation values

p63-CreERT2 mice and determined that essentially all arising $\mathrm{Krt} 5^{\text {pos }}$ cells were lineage labelled (Fig. 1a,b and Supplementary Fig. 1a). Uninjured mice possessed p63-lineage-labelled cells scattered throughout airways, comprising $<0.01 \%$ of all lung epithelial cells (Supplementary Fig. 1b,c). These rare cells must possess remarkable b

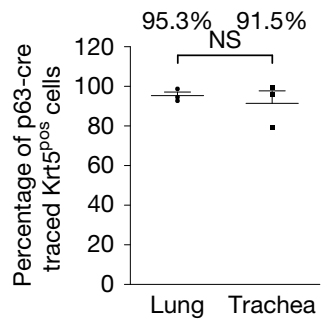

e
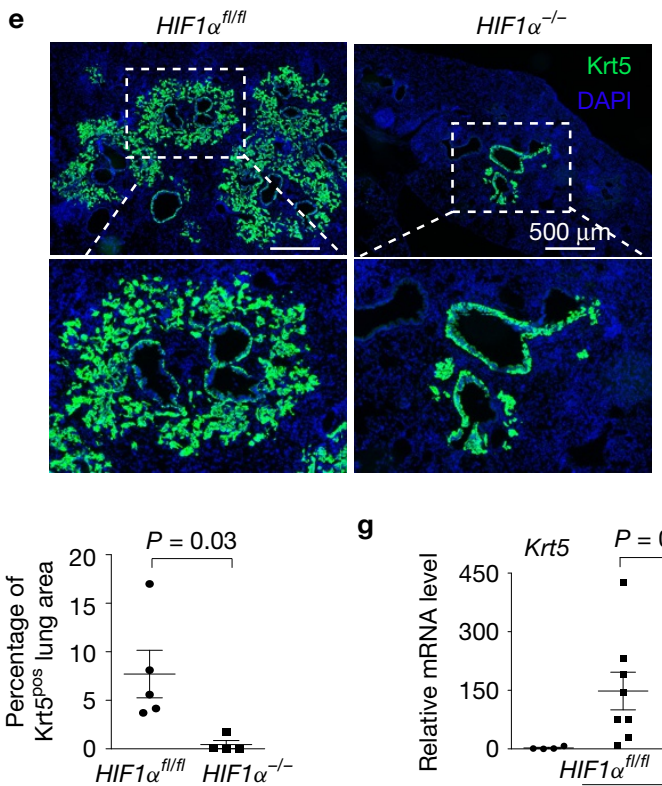

g
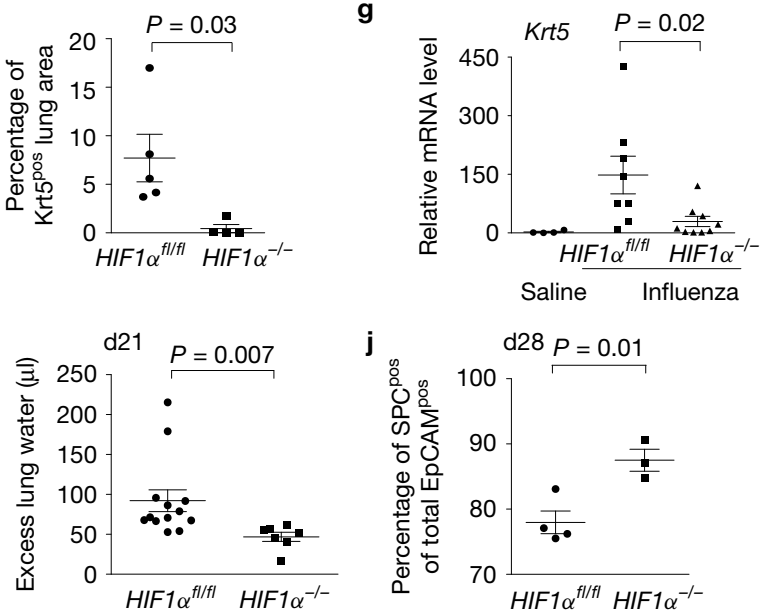

obtained by pulse oximetry are greater in $H I F l \alpha^{-/-}$at the indicated times. (i) $H I F l \alpha^{-/-}$mice exhibit less excess lung water after influenza, indicative of improved barrier function. Data for $\mathbf{h}$ and $\mathbf{i}$ are mean \pm s.e.m., $n=7$ HIF $1 \alpha^{-/-}, n=14$ wild-type (2 Shh-Cre ${ }^{\text {neg }}, 12$ C57BL6) or $n=13$ wildtype for i (2 Shh-Cre ${ }^{\text {neg }}, 11$ C57BL6) from two independent experiments. (j) AEC2 recovery/regeneration is improved in $H I F l \alpha^{-/-}$as judged by intracellular FACS for SPC ${ }^{\text {pos }}$ cells as a fraction of total EpCAM ${ }^{\text {pos }}$ cells. The percentage of EpCAM ${ }^{\text {pos }}$ live cells is unchanged in $H I F l \alpha^{-/-}$and wild-type mice. Data are mean \pm s.e.m., $n=3 \mathrm{HIFl} \alpha^{-1-}, n=4$ wild-type mice from 2 independent experiments. Analysis is 11 days post-infection unless otherwise indicated. All $P$ values were derived by unpaired twotailed Student's $t$-test, except in $\mathbf{h}$, derived by Mann-Whitney. NS, not significant. Unprocessed original scans of blots are shown in Supplementary Fig. 8.

resistance to infection as well as migratory and proliferative capacity. However, the scarcity of $\mathrm{p} 63^{\text {pos }}$ LNEPs and their infrequent contribution to AEC2 regeneration (Supplementary Fig. 1a) suggest that additional $\mathrm{p} 63^{\text {neg }}$ Sox $2^{\text {pos }}$ LNEPs are a likely source of previously observed AEC2 differentiation following LNEP transplantation post 
influenza injury ${ }^{2}$. To begin to dissect these possibilities we considered signals that impact LNEP expansion in vivo.

\section{Epithelial HIF $1 \alpha$ is required for $\mathrm{Krt5}^{\mathrm{pos}}$ cell expansion post influenza injury}

To explore mechanisms for $\mathrm{p} 63^{\text {pos }}$ LNEP expansion during influenza infection, we examined the role of hypoxia, a likely consequence of extensive epithelial necrosis. $\mathrm{Krt} 5^{\text {pos }}$ areas were found exclusively in hypoxic regions identified by immunostaining of pimonidazole (hypoxyprobe) (Fig. 1c). HIF1 $\alpha$, a key mediator of cell responses to hypoxia, accumulated in the whole lung lysates 11 days post infection (Fig. 1d), confirming that regions of the lung are hypoxic.

We then explored the functional relationship between hypoxia and Krt5 ${ }^{\text {pos }}$ cell expansion/activation. Pan epithelial deletion of HIF1 $\alpha$ (Shh-Cre; HIF $1 \alpha^{f l / f l}$, designated as $H I F 1 \alpha^{-/-}$) results in viable mice, born at expected Mendelian ratios, and displaying no overt defects, while epithelial HIF1 $\alpha$ messenger RNA was decreased by $99 \%$ (Supplementary Fig. 1d-f). Eleven days after influenza injury, the $H I F 1 \alpha^{-/-}$ mice showed a dramatic decrease in alveolar $\mathrm{Krt} 5^{\text {pos }}$ cell expansion (Fig. 1e-f), as well as Krt5 protein (Supplementary Fig. 2a) and mRNA (Fig. 1g) levels, with no inhibition of airway Krt5 ${ }^{\text {pos }}$ expansion. Expansion of LNEPs was attenuated, demonstrated by a lack of integrin $\beta 4^{\text {pos }}$ and p63 ${ }^{\text {pos }}$ expanding cells (Supplementary Fig. 2b,c). We next addressed the possibility that $H I F 1 \alpha^{-/-}$mice were less injured. Regardless of HIF1 $\alpha$ deletion, viral protein was detected on day 4 and cleared by day 11 (Supplementary Fig. 2d). Both groups lost 20-30\% weight (Supplementary Fig. 2e) and no difference was observed in the inflammatory cell counts or protein levels in bronchoalveolar lavage (Supplementary Fig. 2f,g). Comparable large regions depleted of alveolar epithelial cells developed in all mice (Supplementary Fig. 2h,i). $H I F 1 \alpha^{-1-}$ mice are therefore injured at similar levels as wild-type mice, but lack alveolar Krt5 ${ }^{\text {pos }}$ cell expansion.

\section{HIF $1 \alpha$ deletion promotes functional regeneration}

While injury levels were equivalent, epithelial $H I F 1 \alpha^{-/-}$mice regained weight more rapidly after the acute injury phase (Supplementary Fig. 2j). Remarkably, arterial oxygen saturation in $H I F 1 \alpha^{-/-}$ mice also recovered more quickly, and barrier function was likewise improved as judged by less interstitial and/or alveolar edema (Fig. 1h,i and Supplementary Fig. 2k). Furthermore, AEC2s constituted a higher fraction of the total epithelium in $H I F 1 \alpha^{-/-}$mice after recovery (Fig. 1j). To address the source of the newly generated AEC2s, we deleted HIF $1 \alpha$ specifically in Sox $2^{\text {pos }}$ cells, including both $\mathrm{p} 63^{\text {pos }}$ and p63 ${ }^{\text {neg }}$ LNEPs but not AEC2s, with Sox2-CreERT2/tdTomato mice (Fig. 2a). HIF1 $\alpha$ deletion from the Sox $2^{\text {pos }}$ lineage resulted in strong attenuation of alveolar $\mathrm{Krt} 5^{\text {pos }}$ cell expansion after infection (Fig. 2b), similar to global epithelial HIF1 $\alpha$ deletion (Fig. 1e). Instead, the majority of Sox2-traced alveolar cells exhibited AEC2 differentiation, as judged by location and SPC expression (Fig. 2a,c). Sox2-labelled AEC2s were much more proliferative than either endogenous AEC2s in the same injured regions or AEC2s in influenza-injured lungs of mice with HIF1 $\alpha$ deletion specifically in pre-existing AEC2s (Fig. 2d,e). While HIF1 $\alpha$-driven $\mathrm{Krt5}^{\text {pos }}$ expansion supports some degree of functional recovery ${ }^{7}$, HIF $1 \alpha$ deletion substantially improves lung function by re-directing airway progenitor responses toward rapid AEC2 expansion and migration, bypassing basal-like metaplasia entirely. Given the impact of HIF1 $\alpha$ on the quality of epithelial recovery, we explored the mechanistic link between hypoxia and $\mathrm{Krt}^{\mathrm{pos}}$ cell expansion.

\section{Hypoxia promotes Notch signalling and Krt5 expression in a HIF $1 \alpha$-dependent manner}

We validated that submersion cultures are hypoxic, as previously reported $^{12}$, in comparison with air/liquid interface demonstrated by hypoxyprobe staining and elevation of HIF1 $\alpha$ targets (Fig. 3a). Hypoxia also promoted Notch target and Krt5 mRNA expression (Fig. 3a), suggesting that HIF1 $\alpha$ regulates Notch activity. Primary LNEP-enriched cells (EpCAM ${ }^{\text {pos }}$ integrin $\beta 4^{\text {pos }}$ ) expressed negligible $\mathrm{Kr} 5 \mathrm{~b}$ but its expression dramatically increased during the first 7-10 days in vitro, and continued over several passages alongside other basal cell genes (Fig. 3b). In contrast, LNEPs isolated from $H I F 1 \alpha^{-/-}$ mice expressed lower levels of basal cytokeratins and Notch target genes, indicating that HIF $1 \alpha$ is required for Notch activity. HIF $1 \alpha^{-/-}$ LNEPs formed fewer and smaller colonies (Fig. 3e and Supplementary Fig. 3a,b) but demonstrate increased SPC expression (Fig. 3c,d and Supplementary Fig. 3c) consistent with the suppressive effects of Notch signalling on alveolar differentiation ${ }^{2,8,13,14}$.

Because HIF1 $\alpha$ loss had no effect on total NICD1 level (Fig. 3f), we tested whether HIF $1 \alpha$ could promote NICD DNA binding via chromatin immunoprecipitation (ChIP) experiments. Both HIF1 $\alpha$ and NICD1 associated with CSL-binding sites (CBE) and HIF1 $\alpha$ binding sites (HRE) on Krt5, Heyl and Hes 5 promoters (Fig. $3 \mathrm{~g}$ and Supplementary Fig. 3d). NICD1 binding on DNA was abolished by HIF1 $\alpha$ deletion. Multiple binding sites on the promoters were tested and all showed similar results.

To confirm Notch signalling as a driver of LNEP expansion, we analysed the transcriptomes of highly purified quiescent LNEPs (EpCAM $^{\text {pos }} \beta 4^{\text {pos }} \mathrm{CC} 10^{\text {neg }}$ FoxJ $1^{\text {neg }}$ ) (Supplementary Fig. 3e) and activated LNEPs (Krt5-CreERT2 traced cells 17 days post infection). Activated LNEPs showed high induction of Notch and HIF1 $\alpha$ target genes and Krt5 expression (Fig. 3h). The loss of Notch activity in $\mathrm{HIF} 1 \alpha$-deficient cells correlates directly with the failure of $\mathrm{Krt5} 5^{\text {pos }}$ cell expansion in vivo in $H I F 1 \alpha^{-/-}$mice. Thus, injury-induced hypoxia, through HIF1 $\alpha$, drives Notch activity by empowering NICD binding on Notch target gene promoters, and promoting LNEP differentiation towards Krt5 ${ }^{\text {pos }}$ basal-like cells. Of note, HIF $1 \alpha$ deletion had little apparent effect on Notch regulation of airway epithelium $^{15-17}$ (Supplementary Fig. 3f,g).

\section{Stabilization of $\beta$-catenin promotes LNEP differentiation towards AEC2s}

Because Wnt signalling is important for the development of alveolar epithelium ${ }^{18-21}$, we tested whether activating Wnt could promote LNEP differentiation into AEC2s in vivo, similar to HIF1 $\alpha$ deletion. We crossed Sox2-CreERT2/tdTomato mice with $\beta$-catenin gainof-function mice $\left(\beta\right.$-catenin $\left.{ }^{\text {loxEx3}}\right)$ to stabilize $\beta$-catenin in Sox $2^{\text {pos }}$ LNEPs. $\beta$-catenin stabilization in Sox 2 -traced cells (tdTomato $^{\text {pos }}$ ) led to ectopic SPC expression in the airways even without challenge, demonstrating that Wnt activity alone could induce a fate change even in quiescent adult progenitors (Supplementary Fig. 4a). Expression of SPC and expression of Scgb3a2 (a highly specific club cell marker) (2,23 $^{22}$ were largely mutually exclusive, and previous reports activating 

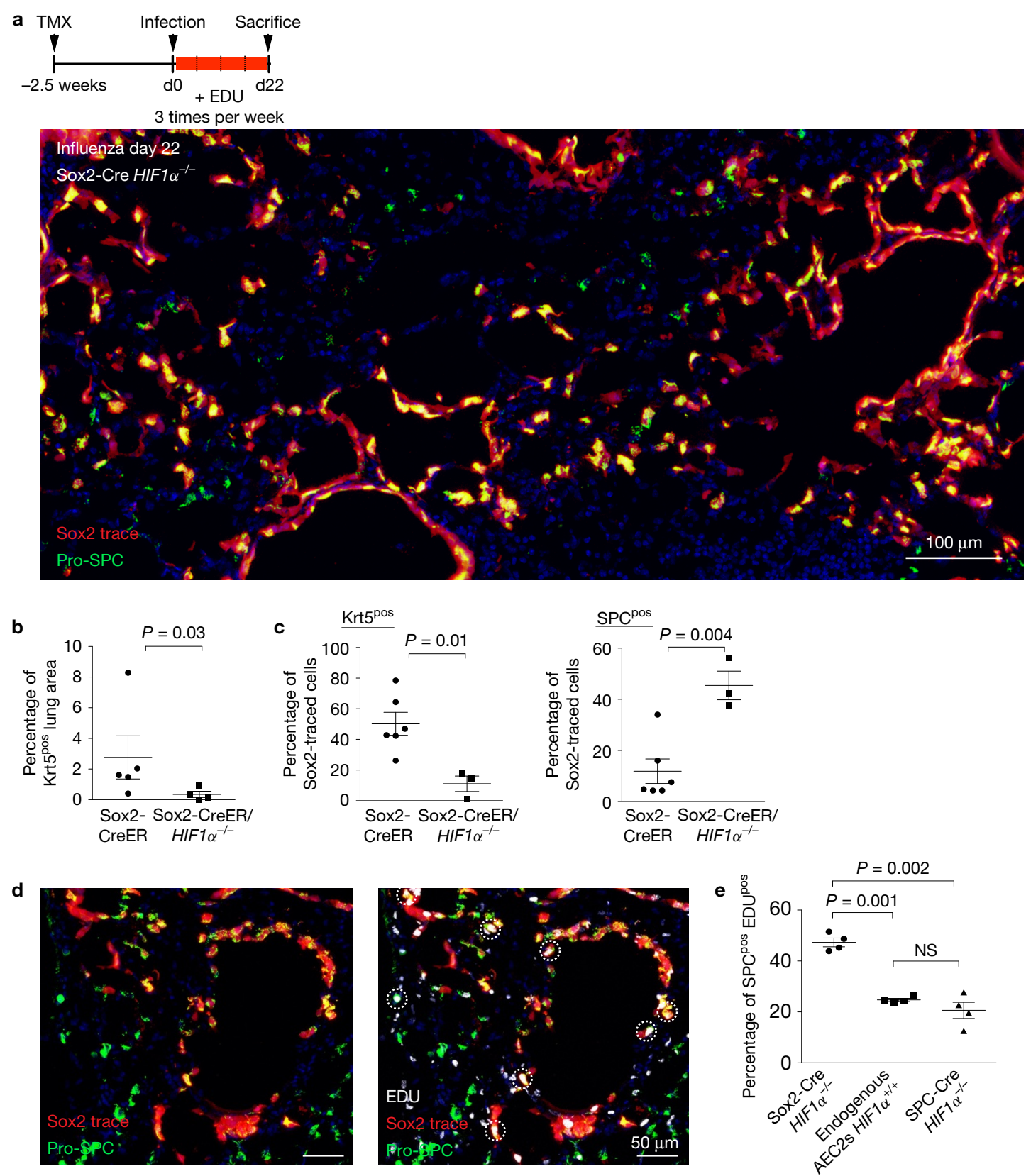

Figure 2 HIF $1 \alpha$ deletion in Sox $2^{\text {pos }}$ LNEPs promotes regeneration of AEC2s. (a) HIF1 $\alpha$ deletion with Sox2-CreERT2 prior to infection results in abundant SPC ${ }^{\text {pos }}$ (green) traced cells by 22 days post-influenza. (b) HIF1 $\alpha$ deletion in Sox $2^{\text {pos }}$ cells blocks Krt5 expansion as in pan-epithelial deletion (Fig. 1f). (c) Sox2 $2^{\text {pos }}$ cell fate is redirected from Krt5 ${ }^{\text {pos }}$ to SPC ${ }^{\text {pos }}$ cells as determined by relative fractions of total alveolar Sox2-traced cells. (d) Newly generated Sox2-traced, HIFl $\alpha^{-1-}$ AEC2s incorporate more EDU (white) than nearby, endogenous AEC2s or AEC2s with HIF1 $\alpha$ deletion (utilizing

$\beta$-catenin in mature club cells resulted in no increase in SPC expression $^{24}$, indicating that most newly arising SPC $^{\text {pos }}$ cells were derived from Sox $2^{\text {pos }}$ LNEPs and not mature club cells. After infection, Sox2traced cells expanded and differentiated primarily into $\mathrm{Krt} 5^{\text {pos }}$ cells, whereas $\beta$-catenin stabilization dramatically switched differentiation from $\mathrm{Krt}^{\text {pos }}$ to $\mathrm{SPC}^{\text {pos }}$, leading to AEC2 expansion (Fig. 4a,b).

We further explored the impact of Notch and Wnt signalling on LNEP differentiation in vitro. Treatment with the GSK3 $\beta$ inhibitor
SPC-CreERT2 mice). (e) Quantification of d, as the fraction of total traced or untraced $\mathrm{SPC}^{\mathrm{pos}}$ cells that are also EDU ${ }^{\text {pos }}$. Data are mean \pm s.e.m.; $n=4$ Sox2-CreERT2/HIF1 $\alpha^{-/-}$in $\mathbf{b}, \mathbf{e} ; n=5$ Sox2-CreERT2/HIF1 $\alpha^{+/+}$in $\mathbf{b}$;

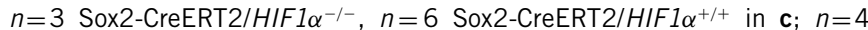
SPC-CreERT2/HIF $1 \alpha^{-/-}$in e. Sox2-CreERT2/HIF $1 \alpha^{+/+}$mice are denoted as 'Sox2-CreER' on the axis. Analysis is 22 days post-infection. $P$ values derived by Mann Whitney in $\mathbf{b}$ and unpaired $t$-test with Welch's correction in $\mathbf{c}, \mathbf{e}$.

CHIR99021 inhibited Notch and hypoxia target gene expression while activating Wnt target gene Axin2 expression (Fig. 4c), indicating that Wnt activity antagonizes Notch and hypoxia signalling, favouring AEC2 differentiation of LNEPs (Fig. 4d). ChIP experiments indicated that the inhibition occurs by prevention of NICD1 and HIF1 $\alpha$ association on target promoters, without affecting NICD1 protein level (Fig. 4e,f and Supplementary Fig. 4b). We established clonal cultures of fluorescently tagged LNEPs and observed multiple instances of $\mathrm{Krt}^{\mathrm{pos}}$ 
a
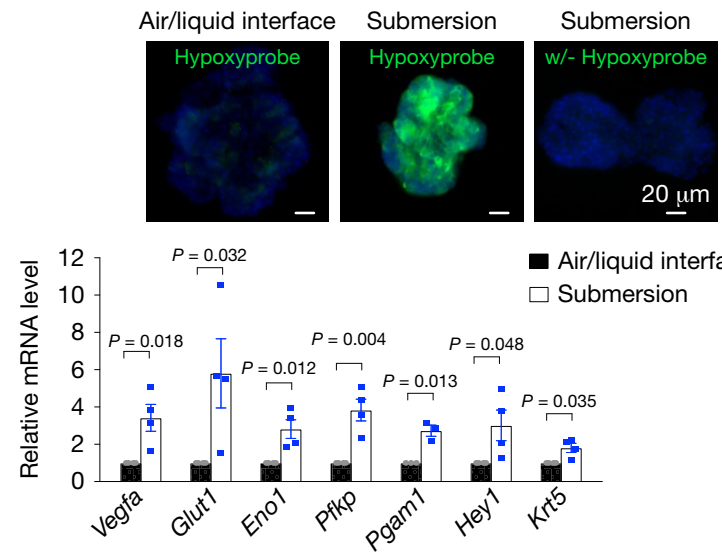

WT $\square H I F 1 \alpha^{-/-}$

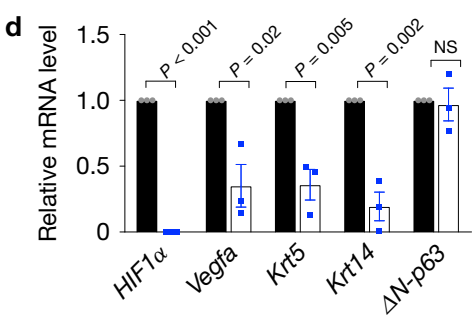

f

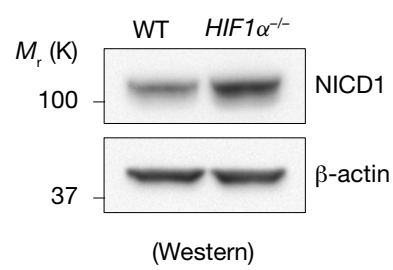

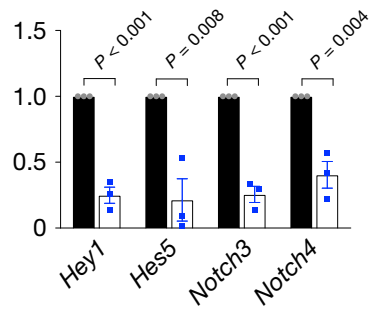

9

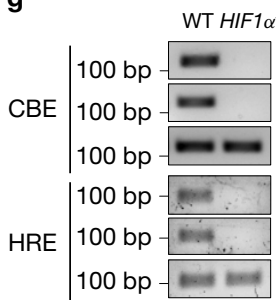

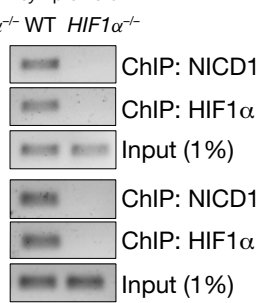

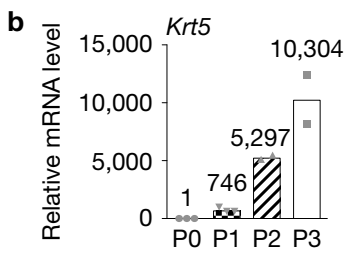

C

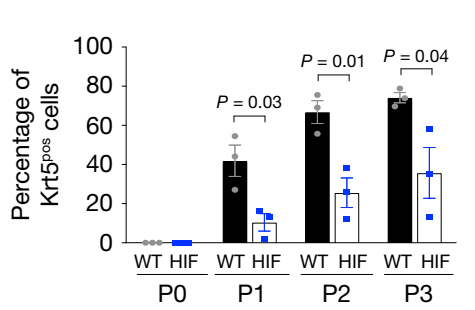

e
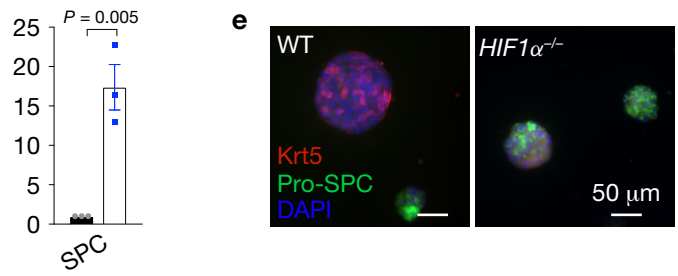

h

\begin{tabular}{|l|r|r|}
\hline & $\begin{array}{c}\text { Norm } \\
\text { LNEPs }\end{array}$ & $\begin{array}{c}\text { Activated } \\
\text { LNEPs }\end{array}$ \\
\hline Krt5 & 0.00 & 25.84 \\
\hline Hey1 & 0.68 & 17.74 \\
\hline Eno1 & 8.36 & 28.78 \\
\hline Pfkp & 4.17 & 16.84 \\
\hline Pgam1 & 3.36 & 7.44 \\
\hline Glut1 & 12.90 & 32.62 \\
\hline \multicolumn{3}{|c|}{ (FPKM) } \\
\hline
\end{tabular}

Figure 3 HIF $1 \alpha$ drives Notch signalling in vitro and in vivo. (a) LNEPs in submersion culture are hypoxic (hypoxyprobe, green) in comparison with LNEPs in air/liquid interface culture. Submersion results in upregulation of hypoxia and Notch target genes as well as Krt5. Data are mean \pm s.e.m., $n=4$ independent experiments. (b) Krt5 and Vegfa mRNA increase with time (passage) in cultured LNEPs. P0, primary cells, P1-P3, passages, $n=2$ independent experiments. (c) HIF $1 \alpha$-deficient LNEPS exhibit reduced Krt5 and increased SPC expression as measured by the percentage of positive cells in culture. WT, wild-type; HIF, HIFl $\alpha^{-/-}$. (d) Expression levels of basal cytokeratins and Notch target genes are similarly reduced in HIFI $\alpha^{-/-}$LNEPS, in contrast to elevated SPC expression. In c,d, data are mean \pm s.e.m. from $n=3$ independent experiments. (e) Representative images of P1 colonies (primary cells cultured for 1 week) showing smaller colony size, reduced Krt5 and increased SPC expression in HIF1 $\alpha$-deficient LNEPs culture. (f) Representative blots of three independent experiments showing that the NICD1 protein level is not affected by HIF $1 \alpha$ deletion in cultured LNEPS. (g) HIF $1 \alpha$ and NICD1 associate with both CSL-binding element (CBE) and HIF-responsive element (HRE) on the Krt5 and Heyl promoters by ChIP. HIF $1 \alpha$ deletion completely prevents NICD1 association on promoter DNA. See qPCR quantification as well as Hes5 promoter analysis from three independent experiments in Supplementary Fig. 3d. (h) RNA-Seq results showing that activated LNEPs (Krt5-CreERT2 traced cells 17 days post infection) have higher Krt5, Notch and hypoxia target gene expression as compared with LNEPs from uninfected mice ( $n=5$ mice per group). $P$ values derived by unpaired two-tailed Student's $t$-test, except in a, derived by one-sample $t$-test. Unprocessed original scans of blots are shown in Supplementary Fig. 8. and $\mathrm{SPC}^{\mathrm{pos}}$ cells in a single-colour clone, indicating that both cell types could arise from a single cell (Fig. 4g and Supplementary Fig. 4c), although some colonies were homogeneously $\mathrm{Krt} 5^{\mathrm{pos}}$ or SPC ${ }^{\mathrm{pos}}$. Likewise, $\mathrm{p} 63^{\text {neg }}$ LNEPs isolated from tamoxifen-treated p63-CreERT2 mice demonstrated $\sim 10 \%$ recombination after one culture passage following addition of 4OHT (Supplementary Fig. 4d), confirming differentiation of $p 63^{\text {neg }}$ to $p 63^{\text {pos }}$ cells. Most $p 63^{\text {neg }}$ LNEPs in culture expand as either undifferentiated cells or as $\mathrm{SPC}^{\mathrm{pos}}$ cells, the latter significantly enhanced by Wnt activation (Fig. 4d and Supplementary Fig. 4d). Therefore, stabilization of $\beta$-catenin blocks Notch and hypoxia signalling, attenuates Krt5 activation, and promotes AEC2 expansion after influenza infection. Interestingly, while $\mathrm{Krt} 5^{\text {pos }}$ cells in vitro arise from $\mathrm{p} 63^{\text {neg }}$ LNEPs (Supplementary Fig. $4 \mathrm{~d}$ ), following influenza infection, activated $\mathrm{Krt} 5^{\mathrm{pos}}$ cells in vivo are derived virtually entirely from rare LNEPs already expressing p63 (Fig. 1a), suggesting that $\mathrm{p} 63^{\text {neg }}$ LNEPs in vivo are intrinsically slower to differentiate to $\mathrm{Krt5} 5^{\text {pos }}$ cells or rendered dysfunctional by H1N1 infection.

\section{Deleting HIF $1 \alpha$ or stabilizing $\beta$-catenin does not alter LNEP differentiation after full Notch/Krt5 activation.}

To investigate the possibility of LNEP transdifferentiation to AEC2s after Krt5 activation, we crossed Krt5-CreERT2; tdTomato mice with either $H I F 1 \alpha^{f l / f l}$ or $\beta$-catenin ${ }^{\operatorname{loxEx} 3}$ mice and treated with tamoxifen 7-10 days post infection to delete HIF1 $\alpha$ or stabilize $\beta$-catenin specifically in activated LNEPs. Neither blocking HIF1 $\alpha$ nor activating Wnt signalling altered Krt5 ${ }^{\text {pos }}$ cell fate: Krt5 traced cells did not give 
a

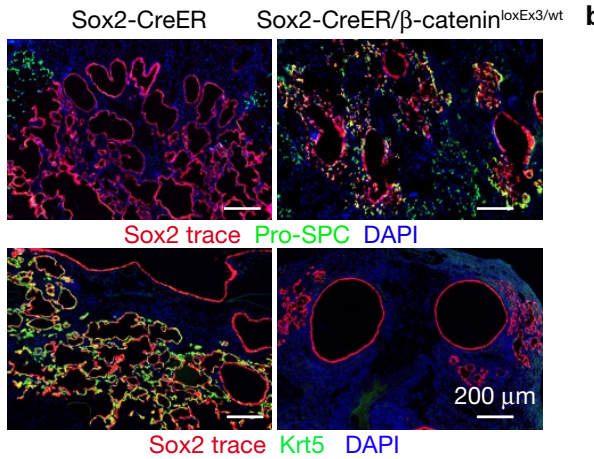

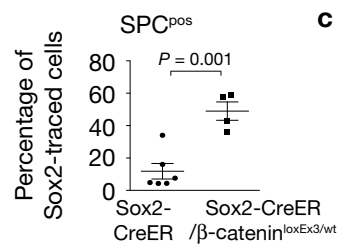

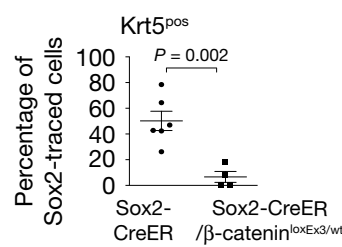

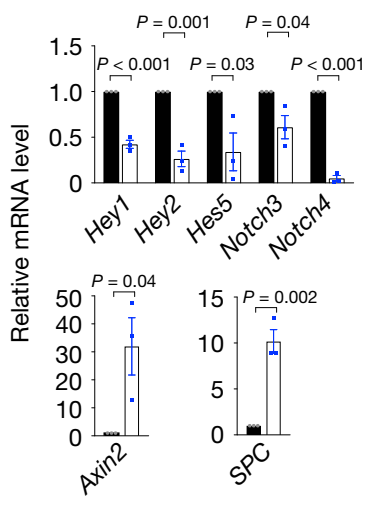
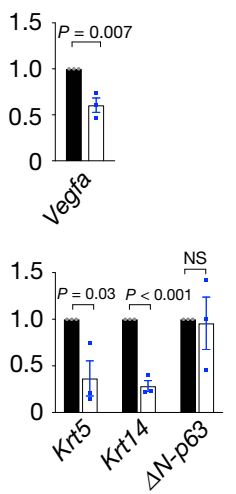

d

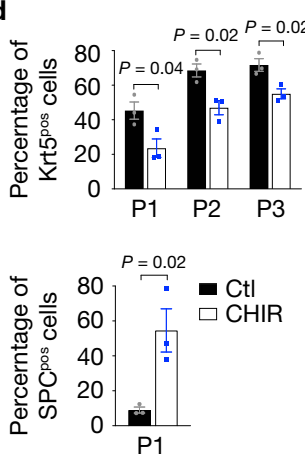

e Krt5 promoter Hey1 promoter

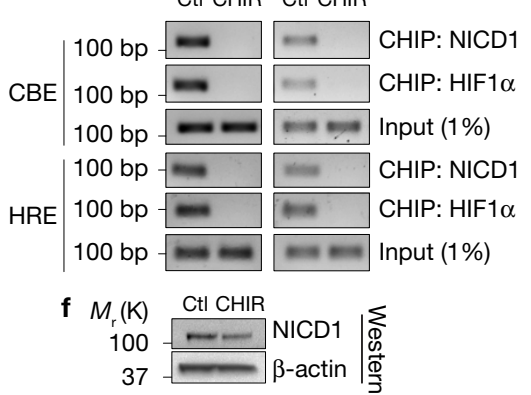

g

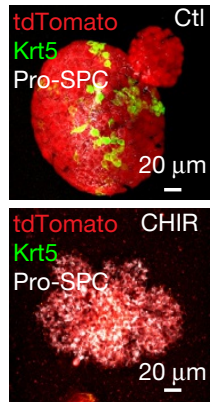

h

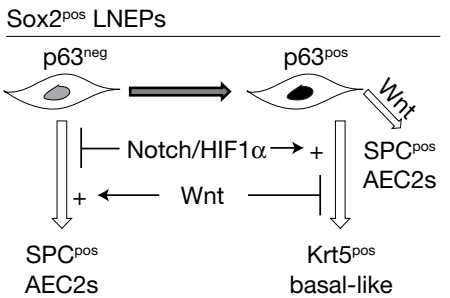

Figure 4 Stabilization of $\beta$-catenin promotes LNEP differentiation towards an alveolar fate. (a) Krt5 ${ }^{\text {pos }}$ cells and to a lesser degree SPC ${ }^{\text {pos }}$ cells are traced by Sox2-CreERT2 in response to influenza injury (left). Stabilization of $\beta$-catenin in Sox2-expressing cells prior to injury by tamoxifen administration results in a dramatic increase in traced SPC ${ }^{\text {pos }}$ cells and concurrent decrease in Krt5 ${ }^{\text {pos }}$ cells (right). (b) Quantification of the percentage of the Sox2-CreERT2 traced cells expressing Krt5 (bottom) or SPC (top) in injured alveolar areas. Data are mean \pm s.e.m., $n=4$ with $\beta$-catenin stabilization, $n=6$ without (same mice from Fig. 2c) from two independent experiments. (c) CHIR99021 treatment phenocopies HIF $1 \alpha$ deletion (see Fig. 3d), reducing expression of Notch and HIFl $\alpha$ target genes and basal cytokeratins while increasing SPC and Axin2 by qPCR analysis. (d) Wnt agonism of LNEPs in vitro decreases the frequency (\%) of $\mathrm{Krt} 5^{\mathrm{pos}}$ cells with a concurrent increase of $\mathrm{SPC}^{\mathrm{pos}}$ cells in cytospin analysis. In $\mathbf{c}, \mathbf{d}$, data are mean \pm s.e.m. from $n=3$ independent experiments. (e) CHIR99021 treatment further mirrors HIFl $\alpha$ deletion in preventing association of NICD1 and HIF1 $\alpha$ with CBE and HRE sites on the Krt5 and Heyl promoters as determined by ChIP. See qPCR

rise to AEC2s two weeks post tamoxifen, retaining Krt5 expression and Notch activity (Supplementary Fig. 5). In the context of severe influenza infection, it appears that once the Notch/Krt5 program is activated, signals that redirect initial LNEP differentiation become ineffective in inducing transdifferentiation of Krt5-committed cells.

\section{Krt5 ${ }^{\text {pos }}$ expansion represents a common response to major lung injury in both mice and humans}

As hyperactive Notch/Krt5 $5^{\text {pos }}$ cysts exist in lungs from patients with pulmonary fibrosis ${ }^{2}$, we examined whether the expansion of Krt5 $5^{\text {pos }}$ cells is a common response to major injury. In lung tissue obtained from six patients who succumbed to $\mathrm{H} 1 \mathrm{~N} 1$ influenza A virus there was widespread destruction of parenchymal cells and three patient samples evidenced an extensive regenerative response (Fig. 5a,b). Large areas were devoid of SPC ${ }^{\text {pos }}$ AEC2s and comprised instead of $\mathrm{Krt}^{\text {pos }}$ quantification as well as Hes 5 promoter analysis from three independent experiments in Supplementary Fig. 4b. (f) Representative blots of three independent experiments showing that the NICD1 protein level is not affected by $\beta$-catenin stabilization. (g) LNEPs isolated from ubGFP (GFPpos) and $\mathrm{mTmG}$ (tdTomato ${ }^{\text {pos }}$ ) were mixed in equal ratios and cultured resulting in largely single-colour colonies (clones). Representative images of tdTomatopos colonies demonstrate SPC ${ }^{\text {pos }}$ and $\mathrm{Krt}^{\text {pos }}$ cells in single clones whereas most colonies exhibit uniform SPC expression when treated with GSK3 $\beta$ inhibitor CHIR99021 $(2 \mathrm{nM})$. Krt5 is pseudocoloured green for visualization since these colonies were uniformly tdTomato ${ }^{\text {pos }}$. (h) Schematic model of Sox2 ${ }^{\text {pos }}$ LNEP activation in mouse lungs in response to severe injury. HIF $1 \alpha /$ Notch promotes $\mathrm{Krt}^{\mathrm{pos}}$ basal-like cell expansion from $\mathrm{p} 63^{\text {pos }} \mathrm{LNEPs}$. Wnt/ $\beta$-catenin signalling antagonizes hypoxia and Notch signalling and promotes AEC2 expansion from p63 ${ }^{\text {neg }}$ LNEPs. LNEPs that upregulate p63 can still respond to Wnt signals to generate AEC2s (see Supplementary Fig. 4 d). $P$ values were derived by unpaired two-tailed Student's $t$-test. Unprocessed original scans of blots are shown in Supplementary Fig. 8.

cells that appear flattened and cover much of the alveolar surfaces, reminiscent of LNEP expansion in influenza-infected mouse lungs. In contrast to mice, some areas of affected lung contained numerous $\mathrm{Krt} 5^{\text {pos }}$ cells that were also clearly positive for SPC, suggesting either differentiation of $\mathrm{Krt}^{\mathrm{pos}}$ cells toward AEC2s or transdifferentiation of AEC2s toward basal-like cells. This was paralleled in sections from ARDS (acute respiratory distress syndrome) lungs (Fig. 5c) and by prior observations in scleroderma in which SPC ${ }^{\text {os }} / \mathrm{Krt}^{\text {pos }}$ alveolar cells were observed ${ }^{2}$. We conclude that as in murine influenza injury, human stem/progenitor cells demonstrate robust attempts at re-establishing alveolar epithelial barriers with $\mathrm{Krt} 5^{\text {pos }}$ cells. Although variable, HIF1 $\alpha$ protein was also increased in all idiopathic pulmonary fibrosis (IPF) samples relative to normal lungs, consistent with reports of hypoxia signalling in $\operatorname{IPF}^{25,26}$ (Fig. 5d). To further investigate the relationship between hypoxia and $\mathrm{Krt} 5^{\text {pos }}$ cell expansion in tissue 
a
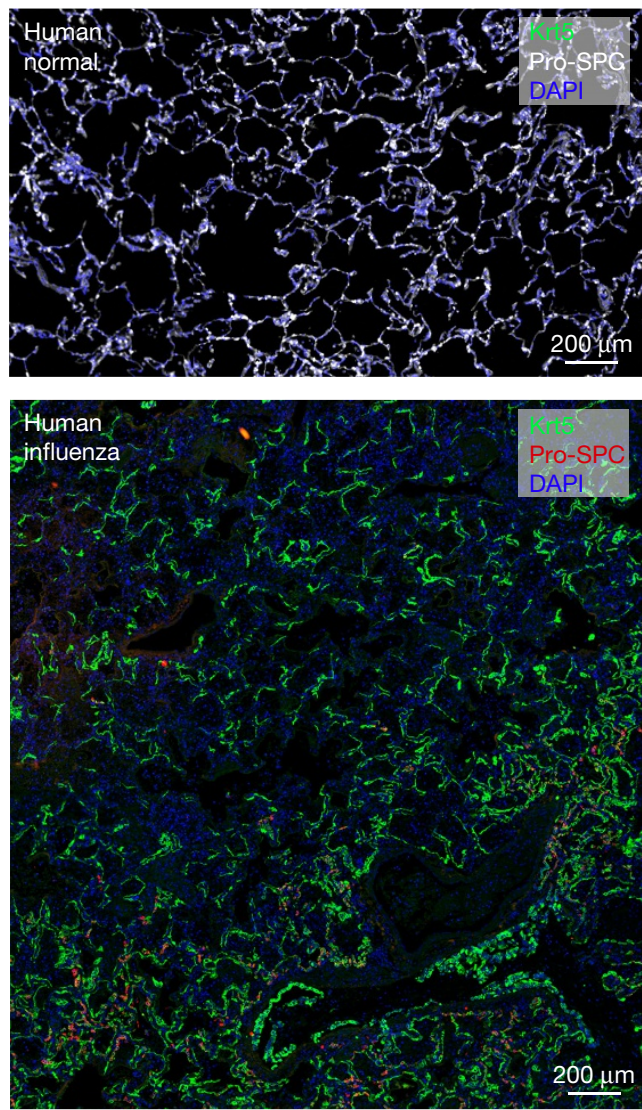
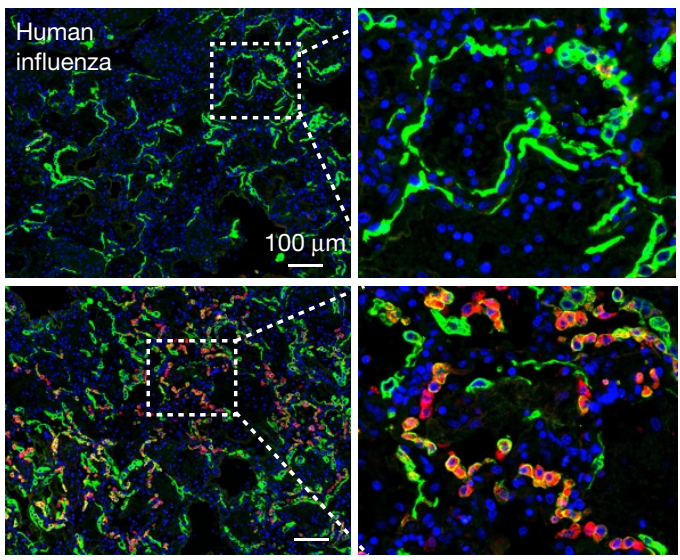

Krt5 Pro-SPC DAPI

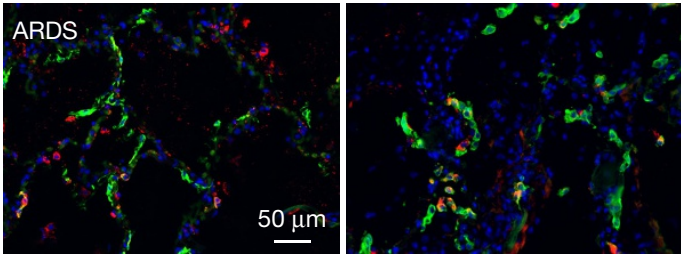

Krt5 Pro-SPC DAPI

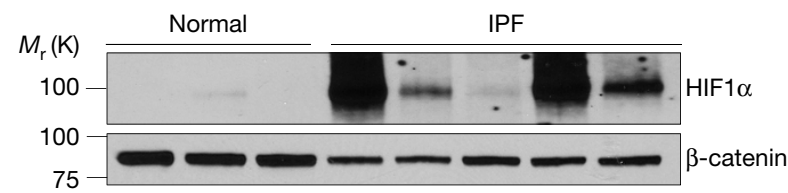

Figure $5 \mathrm{Krt5} 5^{\text {pos }}$ cell expansion is a common response of human lung epithelium to major injury. (a,b) Representative images demonstrating Krt5pos (green) epithelial expansion into the alveolar parenchyma in H1N1 influenzainjured human lungs. Regions of cells co-expressing Krt5 and pro-SPC (red) are also observed (b). (c) Krt5 ${ }^{\text {pos }}$ expansion and Krt5/SPC double-positive cells

repair, we employed single-cell transcriptional profiling of epithelial cells isolated from freshly explanted diseased human lungs.

\section{Evidence that Notch and hypoxia regulate human lung epithelial repair processes}

We performed single-cell RNA-Seq using the Fluidigm $\mathrm{C} 1$ to analyse the transcriptomes of AEC2s (HTII-280 $0^{\text {pos }}$, of which 98\% express SPC) ${ }^{27}$ and basal-enriched cells (integrin $\alpha 6^{6^{\text {pos }}} \mathrm{HTII}-280^{\text {neg }}$, of which 50-70\% express Krt5) (Supplementary Fig. 6). We sequenced between 25 and 72 single cells from normal lungs as well as lungs from patients diagnosed with scleroderma, dyskeratosis congenita and IPF, all fibrotic diseases despite differing etiologies. Principal component analysis (PCA) and whole-genome unsupervised hierarchical clustering clearly separated AEC2s from normal and fibrotic lungs (Supplementary Fig. 7a). The Notch targets HES1 ${ }^{28}$ and SCGB3A2 $2^{22}$ were among the most differentially expressed genes in diseased versus normal populations, corroborating our findings in fibrotic human lungs (Supplementary Table 1). Furthermore, HIF1 $\alpha$ stands out as a top upstream regulator in the diseased AEC2s based on ingenuity pathway analysis (IPA, QIAGEN, www.qiagen.com/ingenuity) (Supplementary Table 2).

We generated a hypoxia-induced gene expression signature by combining three published hypoxia signatures ${ }^{29,30}$ (IPA HIF1 $\alpha$ are also present in ARDS lungs. (d) Representative blot of two independent experiments showing HIF1 $\alpha$ accumulation in the lungs of IPF patients ( $n=9$ IPF, $n=7$ normal in total, $\beta$-catenin as loading control), indicating that hypoxia occurs in IPF lungs. Unprocessed original scans of blots are shown in Supplementary Fig. 8.

pathway). Hierarchical clustering based on the hypoxia signature plus Notch target HES1, KRT5 and AEC2 markers showed three distinct populations: normal AEC2s (Groups I and II), pathologic AEC2s with a hypoxia signature and high HES1 (Group III), and pathologic AEC2s with hypoxia targets and HES1, but decreased surfactants and upregulated basal cytokeratins (Group IV) (Fig. 6a-c and Supplementary Table 3). Adding normal basal-enriched cells (Group V) demonstrated that Group IV was transcriptionally more similar to basal-enriched cells than normal AEC2s. Groups I and II contained nearly all cells isolated from normal lungs that do not clearly segregate based on unbiased clustering, suggesting that the mild hypoxia target expression in Group II may be a technical artefact (Fig. 6d and Supplementary Table 4). Adding 27 AEC2s from IPF lungs placed most IPF cells in Group IV (Supplementary Fig. 7b). Clustering based on the entire transcriptome except the hypoxia signature and lineage markers resulted in nearly identical clustering, validating the hypoxia signature in predictive categorization and demonstrating that meaningful whole-transcriptome (phenotypic) changes occur coordinately with hypoxia signalling (Supplementary Fig. 7c).

The expression pattern transitions from normal cells to basallike cells in Group IV, which is surprisingly similar to normal basalenriched cells (Group V) (Fig. 6e and Supplementary Table 5). Importantly, while Group IV cells develop a basal-like transcriptome 
a

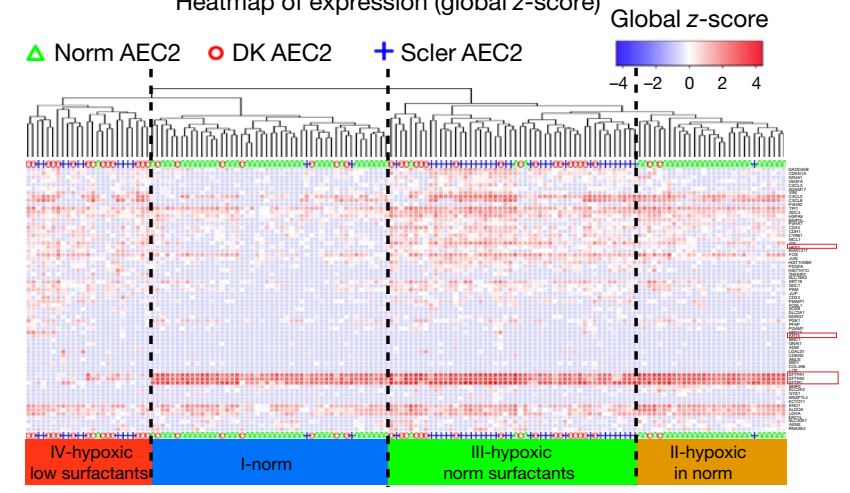

b

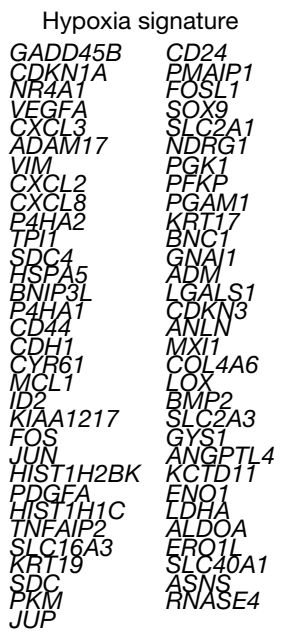

C

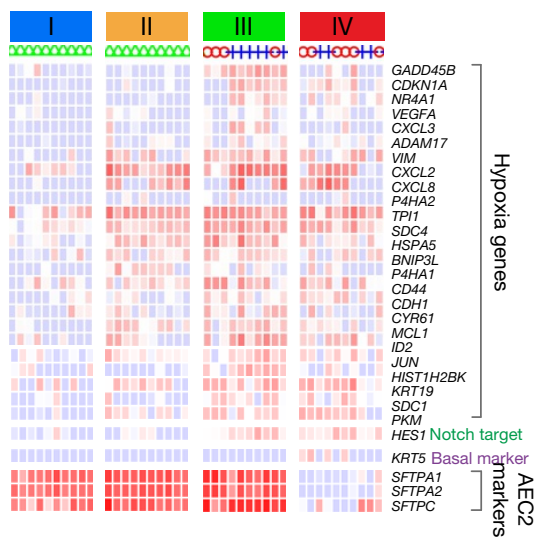

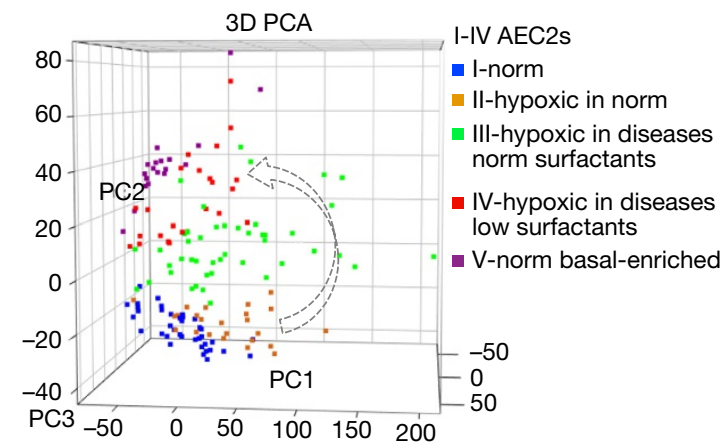

e

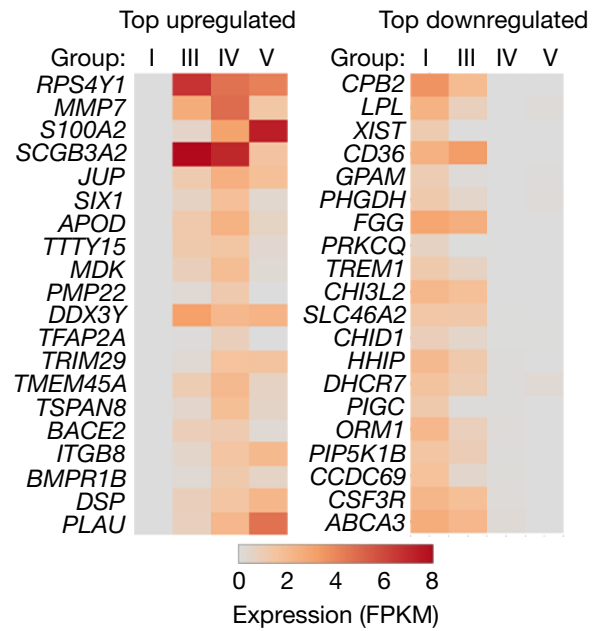

Figure 6 Single-cell RNA-Seq analysis of primary human lung epithelial cells from normal and fibrotic lungs indicates hypoxia/Notch signalling promotes AEC2s transdifferentiation towards basal-like cells after major injury. (a) Hierarchical clustering of single-cell transcriptomes of AEC2s (HTII-280 ${ }^{\text {pos }}$ ) (columns) isolated from normal (triangles), dyskeratosis congenita (DK) (circles), and scleroderma (crosses) lung explants. Clustering reflects hypoxia signature genes (listed in panel b) plus STFPA1, STFPA2, SFTPC, KRT5 and HES1 (highlighted with red boxes) (rows). Four distinct groups (I-IV) are highlighted in different colours: normal AEC2s (Group I, blue), normal AEC2s with hypoxic gene expression (Group II, yellow), AEC2s from diseases with hypoxia signature and HES1 (Group III, green), and

(Fig. 6d,e), most cells retain significant reads for SFTPC whereas no SFTPC was detected in any of the basal-enriched cells. Furthermore, culture of normal AEC2s resulted in marked upregulation of KRT5 mRNA, which was attenuated by Notch inhibition (Supplementary Fig. 7d). These data support a scenario in which human epithelial progenitors utilize a common injury response pathway characterized by hypoxia/Notch/Krt5 and progressively transdifferentiate to a basallike phenotype in diverse disease and injury settings.

\section{A subset of human alveolar epithelial cells, distal airway cells, and murine LNEPs share a migratory gene expression profile}

Activated murine LNEPs and Group IV hypoxic AEC2s shared 102 upregulated genes and 25 downregulated genes (Fig. 7a and Supplementary Fig. 7e and Supplementary Table 6). IPA analysis of the common upregulated genes indicates that HIF $1 \alpha$ is a top upstream regulator (Supplementary Table 7) and cell movement/invasion and proliferation are the top two processes affected (Supplementary
AEC2s from diseases with hypoxia signature and $H E S 1$, with concurrent loss of surfactants and acquisition of Krt5 (Group IV, red). (b) List of hypoxia signature genes. (c) Representative cells and genes of the four groups. (d) Whole-genome PCA analysis of the four groups plus normal basal-enriched cells (HTII-280 $20^{\text {neg }} \alpha 6^{\text {pos }}$, Group V) demonstrates progressive evolution to basal cell-like expression profiles in diseased AEC2s. (e) The expression pattern of the top 20 up- and downregulated genes derived from ANOVA analysis of Group IV versus I, showing the progressive transition from Group I to Group IV, which is very similar to Group V basal-enriched cells. The average FPKM values of the indicated genes averaged within each group $(\mathrm{I}-\mathrm{V})$ are displayed in the heatmap.

Tables 8,9). Importantly, normal human distal airway basalenriched cells (Group V) appear inherently primed by expression of the motility-linked genes induced by hypoxia/Notch in AEC2s (Supplementary Fig. 7e).

We therefore compared motility of human basal-enriched cells with normal human AEC2s. Basal-enriched cells and activated LNEPs were highly motile in vitro in comparison with AEC2s (Fig. 7b). We further analysed the function of two of these motility genes, $A X L$ and $E P H A 2^{31,32}$, which encode receptor tyrosine kinases. Inhibition of AXL or EPHA2 markedly blocked LNEP migration (Fig. 7c). We conclude that hypoxia/Notch activates a proliferative/migratory program in multiple human cell types, implying a common strategy to replenish epithelial barriers under hypoxic, stressful conditions.

\section{DISCUSSION}

The lung is highly efficient at matching air and blood over its enormous alveolar surface area, ensuring oxygen uptake and 
a

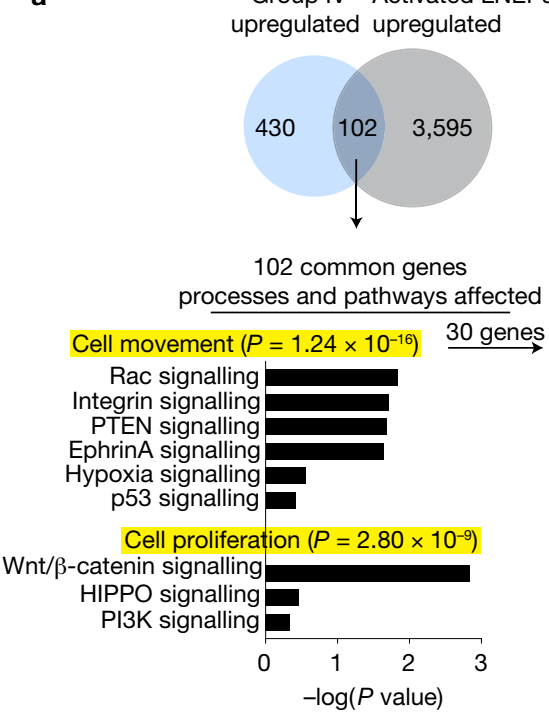

AEC2sLNEPS

$\mathrm{V}$ I IV norm activated

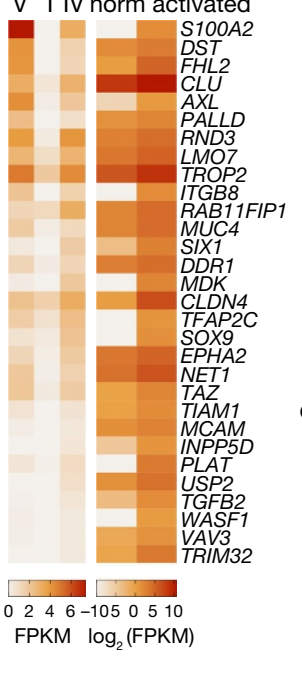

b Group V (basal-enriched cells)

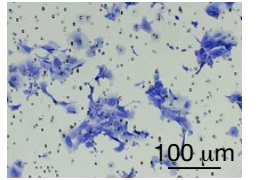

Group I (AEC2s)
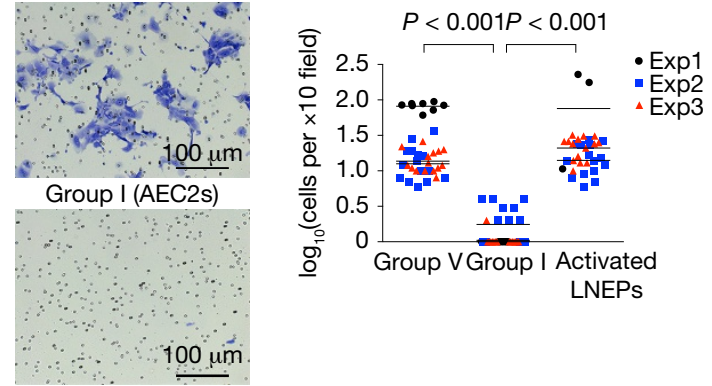

LNEPS
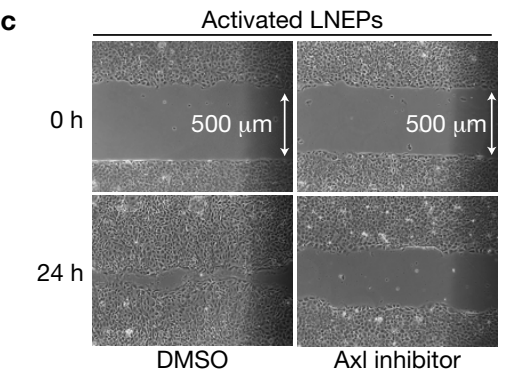

Relative wound area (normalized by $0 \mathrm{~h}$ )

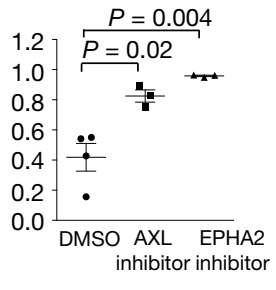

d

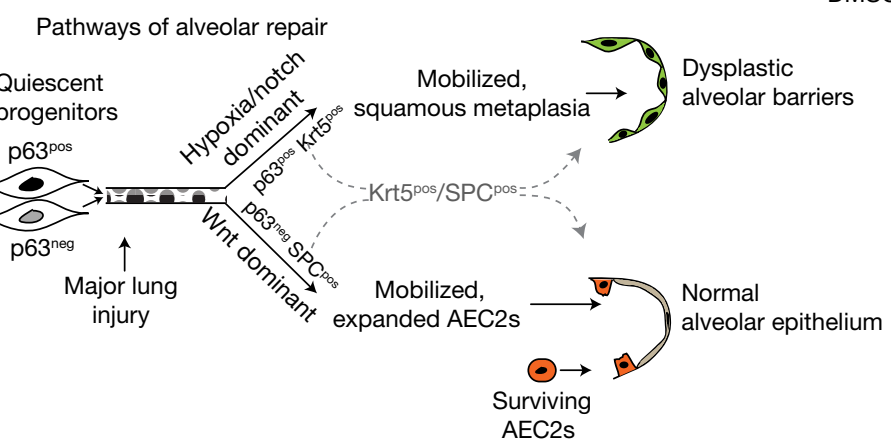

Figure 7 Both human and mouse lung epithelial progenitor cells activate hypoxia/Notch signalling and a motile phenotype in response to major injury. (a) Overlap between mouse activated LNEPs (Krt5 ${ }^{\text {pos }}$ cells versus quiescent LNEPs) and human hypoxic AEC2s (Group IV versus I) identifies pathways strongly implicated in cell movement. Average FPKM values of human cells (Group I, IV and V), and mouse quiescent and activated LNEPs from RNA-Seq are indicated in the heatmap (right) for 30 of these 102 genes directly implicated in migration. IPA analysis of these 102 genes implicates cell movement and cell proliferation as major cellular processes affected, with at least 30 reported to promote cell migration. (b) Human Group $\mathrm{V}$ basal-enriched cells are strikingly motile in a transwell assay, similar to activated LNEPs. Cells that migrated through the pores to the bottom of the insert were stained and quantified. Data are mean \pm s.e.m. from $n=3$ independent experiments. (c) Two tyrosine kinases identified from the common 102 genes, $A X L$ and EPHA2, are functionally important for

delivery. Injury-induced loss of alveolar epithelium leads to alveolar collapse, disrupting critical gas exchange and producing local hypoxia as oxygenated blood is diverted from non-ventilated areas ${ }^{33}$. Regeneration from such injury represents a major challenge given the necessity to reconstitute effective barriers over such a large area. Nonetheless, as judged by the extensive expansion of epithelial progenitor cells throughout the alveoli of flu-injured mouse lungs ${ }^{2,6,7}$ and diversely injured human lungs (Fig. 5a-c), the alveolar epithelium is remarkably regenerative. Whether this regenerative process results in normal or dysplastic repair, however, is dependent on several signalling pathways, and we can now provide additional insights into this process. First, we identify hypoxia itself as a key driver of
LNEP migration. Activated LNEPs treated with AXL- and EPHA2-specific inhibitors ( $3 \mu \mathrm{M}$ R428 and $1 \mu \mathrm{M}$ ALW-II-247) show compromised motility in wound closure assays. Relative wound area at $24 \mathrm{~h}$ as compared with $\mathrm{O} \mathrm{h}$ is graphed as mean \pm s.e.m. from $n=3$ independent experiments. $P$ values were derived by unpaired two-tailed Student's $t$-test. (d) Schematic summary of a common, hypoxia-mediated epithelial progenitor response. Following major lung injury, local hypoxia promotes Notch signalling and a Krt5 ${ }^{\text {pos }}$ remodelling program in quiescent $p 63^{\text {pos }}$ LNEPs via HIF $1 \alpha$, leading to robust migration, squamous metaplasia, and the ultimate formation of dysplastic alveolar barriers. However, Wnt activity prior to Krt5 activation favours $p 63^{\text {neg }}$ LNEP expansion and differentiation toward AEC2s, leading to normal alveolar epithelial repair. The appearance of $\mathrm{Krt} 5^{\mathrm{pos}} / \mathrm{SPC} \mathrm{C}^{\mathrm{pos}}$ cells in several human disease/injury settings further suggests an intermediate response that may result in concurrent dysplastic and appropriate alveolar repair.

Notch signalling critical to activation epithelial stem/progenitor cells (Figs 3, 6 and 7d). Hypoxia has been previously reported to induce Notch activity in vitro ${ }^{34,35}$, and we now provide in vivo evidence that hypoxia drives Notch activity because HIF1 $\alpha$ is essential for NICD DNA binding under hypoxic conditions (Fig. $3 \mathrm{~g}$ and Supplementary Fig. 3d). Secondly, we find that hypoxia/Notch-activated progenitors in both mice and humans upregulate a common set of genes regulating motility and invasion (Fig. 7). Live imaging confirms that activated LNEPs are highly motile within injured lung $^{2}$ as well as in in vitro migration assays (Fig. 7). This phenotype is probably fitted to the migration across large distances through fibrinous environments required for reconstitution of alveolar barriers. That this process in 
lung epithelia is hypoxia driven despite the improved outcome with enhanced AEC2 expansion (Fig. $1 \mathrm{~h}-\mathrm{j}$ ) underscores the fundamental nature of HIF1 $\alpha$-dependent cellular responses to acute hypoxia in all cells and its overall protective effect on survival ${ }^{36}$. Finally, we demonstrate that activation of Wnt or suppression of HIF $1 \alpha$ signalling further empowers non-AEC2 progenitors (Sox $2^{\text {pos }}$ p6 $3^{\text {neg }}$ LNEPs) to contribute to AEC2 recovery and improved lung function. Collectively, these findings highlight both the potential and the signals directing alveolar regenerative programs centred on activation of epithelial progenitors in distal airways.

The ultimate fate of $\mathrm{p} 63^{\text {neg }}$ and $\mathrm{p} 63^{\text {pos }}$ LNEPs appears dependent on both Notch and Wnt signals not only at the level of singlecell differentiation but also at the level of amplification of fated cells by the same signals directing their initial commitment. Thus, in vivo expansion of AEC2s from Sox $2^{\text {pos }}$ cells with HIF1 $\alpha$ deletion or stabilized $\beta$-catenin (Figs 2a and $4 \mathrm{a}$ ) reflects both an initial state change for $\mathrm{p} 63^{\text {neg }} \mathrm{LNEPs}$ resulting in $\mathrm{SPC} \mathrm{C}^{\mathrm{pos}}$ cells and their subsequent expansion into the injured parenchyma. Likewise, p63 $3^{\text {pos }}$ LNEPs are the major responders to HIF1 $\alpha /$ Notch signals producing the entire Krt5 ${ }^{\text {pos }}$ response (Fig. 1a), whereas Wnt suppresses this pathway in favour of p63 ${ }^{\text {neg }}$ LNEP-derived AEC2s (Fig. 4a). The overall outcome of basal-like metaplasia or AEC2 regeneration is largely determined by which pathway dominates the competitive selection between these populations after an initial fate choice, as indicated schematically in Fig. 7d. While Sox $2^{\text {pos }}$ LNEPs are clearly an important contributor to AEC2 regeneration, surviving AEC2s are also proliferative (Fig. 2d) and doubtless participate in alveolar repair ${ }^{1,9,37}$.

Whereas in mice only rare $\mathrm{p}^{63^{\mathrm{pos}}}$ LNEPs account for dramatic Krt5 ${ }^{\text {pos }}$ expansion after influenza (Fig. 1a), human alveoli, after both acute and chronic injury, are re-populated by $\mathrm{Krt}^{\mathrm{p}}{ }^{\mathrm{pos}}$ expansion of several cell types: $\mathrm{SPC}^{\text {pos }} / \mathrm{Krt}^{\text {pos }}$ alveolar cells as well as $\mathrm{p} 63^{\mathrm{pos}}$ LNEP homologues and $\mathrm{p} 63^{\mathrm{pos}} / \mathrm{Krt} 5^{\mathrm{pos}}$ classical basal cells, both prominent in distal human airways ${ }^{2,38}$. Human AEC2s appear more plastic than their murine counterparts as, unlike in mice, human AEC2s rapidly lose SFTPC and express Notch-driven KRT5 when cultured (Supplementary Fig. 7d). Nonetheless, our studies in mice suggest that human lungs may also face the conundrum that, once established after major injury, neither removal of Notch signalling nor Wnt stimulation reverses differentiation down the Krt5 pathway (Supplementary Fig. 5), contributing to a poor functional outcome. It also remains unknown whether there is a true $\mathrm{p} 63^{\text {neg }}$ LNEP in distal human airways capable of contributing to normal alveolar repair. Future studies in humans are needed to both further define the potential for airway progenitors to mobilize a regenerative alveolar program and to elucidate the micro-environmental conditions that favour predominantly AEC2 expansion.

\section{METHODS}

Methods, including statements of data availability and any associated accession codes and references, are available in the online version of this paper.

Note: Supplementary Information is available in the online version of the paper

\section{ACKNOWLEDGEMENTS}

This work was supported by NIH grants RO1 HL128484, UO1 HL111054, UO1 134766, and a sponsored research agreement with Biogen Idec (H.A.C.). A.E.V. was supported by T32 HL007185-36, F32 HL117600-01, and K99 HL131817. M.A.M. and J.E.G. were supported by NHLBI grant R37HL51856 and R37HL57156. J.X. was supported by NIH grants R01 CA112403 and R01 CA193455 and CPRIT grants RP120732-P5 and RP150197. J.M.S. was supported by NIH grant R01 HL084376. We thank M.A.M. and P. Wolters at the UCSF Interstitial Lung Disease Blood and Tissue Repository for procuring non-donor and diseased lung tissues, respectively. We also thank J.E.G. for providing influenza PR8 virus and technical assistance, B. Sennino for hypoxyprobe and technical advice, M. Zhang for CHIR99021 and technical advice, R. Lao of the Institute for Human Genetics core facility, UCSF, for assistance with RNA-sequencing, and D. A. Denison and S. Zaki of the CDC for lung tissue slides obtained from patients with H1N1 influenza.

\section{AUTHOR CONTRIBUTIONS}

Conceptualization: Y.X., H.A.C., A.E.V.; investigation: Y.X., T.K., A.N.B., J.X., D.-K.L., Y.W., V.T., J.R.J., J.M.S., H.A.C., J.E.G., M.A.M., A.E.V.; formal analysis: Y.X., I.H.D., A.E.V.; writing: Y.X., H.A.C., A.E.V.; supervision: H.A.C.; funding acquisition: H.A.C., A.E.V.

\section{COMPETING FINANCIAL INTERESTS}

The authors declare no competing financial interests.

Published online at http://dx.doi.org/10.1038/ncb3580

Reprints and permissions information is available online at www.nature.com/reprints Publisher's note: Springer Nature remains neutral with regard to jurisdictional claims in published maps and institutional affiliations.

1. Desai, T. J., Brownfield, D. G. \& Krasnow, M. A. Alveolar progenitor and stem cells in lung development, renewal and cancer. Nature 507, 190-194 (2014).

2. Vaughan, A. E. et al. Lineage-negative progenitors mobilize to regenerate lung epithelium after major injury. Nature 517, 621-625 (2015).

3. Rawlins, E. L. et al. The role of Scgbla1+ Clara cells in the long-term maintenance and repair of lung airway, but not alveolar, epithelium. Cell Stem Cell 4 , 525-534 (2009).

4. Tata, P. R. et al. Dedifferentiation of committed epithelial cells into stem cells in vivo. Nature 503, 218-223 (2013)

5. Hogan, B. L. et al. Repair and regeneration of the respiratory system: complexity, plasticity, and mechanisms of lung stem cell function. Cell Stem Cell 15, 123-138 (2014).

6. Kumar, P. A. et al. Distal airway stem cells yield alveoli in vitro and during lung regeneration following H1N1 influenza infection. Cell 147, 525-538 (2011).

7. Zuo, W. et al. $\mathrm{p} 63^{+} \mathrm{Krt5} 5^{+}$distal airway stem cells are essential for lung regeneration. Nature 517, 616-620 (2015)

8. Guseh, J. S. et al. Notch signaling promotes airway mucous metaplasia and inhibits alveolar development. Development 136, 1751-1759 (2009).

9. Barkauskas, C. E. et al. Type 2 alveolar cells are stem cells in adult lung. J. Clin. Invest. 123, 3025-3036 (2013).

10. Loosli, C. G. et al. The destruction of type 2 pneumocytes by airborne influenza PR8A virus; its effect on surfactant and lecithin content of the pneumonic lesions of mice. Chest 67, 7S-14S (1975).

11. Ray, S. et al. Rare SOX2+ airway progenitor cells generate KRT5+ cells that repopulate damaged alveolar parenchyma following influenza virus infection. Stem Cell Rep. 7, 817-825 (2016).

12. Gerovac, B. J. et al. Submersion and hypoxia inhibit ciliated cell differentiation in a notch-dependent manner. Am. J. Respir. Cell Mol. Biol. 51, 516-525 (2014).

13. Dang, T. P., Eichenberger, S., Gonzalez, A., Olson, S. \& Carbone, D. P. Constitutive activation of Notch3 inhibits terminal epithelial differentiation in lungs of transgenic mice. Oncogene 22, 1988-1997 (2003).

14. Tsao, P.-N. et al. $\gamma$-secretase activation of notch signaling regulates the balance of proximal and distal fates in progenitor cells of the developing lung. J. Biol. Chem. 283, 29532-29544 (2008).

15. Pardo-Saganta, A. et al. Parent stem cells can serve as niches for their daughter cells. Nature 523, 597-601 (2015).

16. Lafkas, D. et al. Therapeutic antibodies reveal Notch control of transdifferentiation in the adult lung. Nature 528, 127-131 (2015).

17. Morimoto, M., Nishinakamura, R., Saga, Y. \& Kopan, R. Different assemblies of Notch receptors coordinate the distribution of the major bronchial Clara, ciliated and neuroendocrine cells. Development 139, 4365-4373 (2012).

18. Mucenski, M. L. et al. $\beta$-Catenin is required for specification of proximal/distal cell fate during lung morphogenesis. J. Biol. Chem. 278, 40231-40238 (2003).

19. Okubo, T. \& Hogan, B. L. Hyperactive Wnt signaling changes the developmental potential of embryonic lung endoderm. J. Biol. 3, 11 (2004).

20. Hashimoto, S. et al. $\beta$-Catenin-SOX2 signaling regulates the fate of developing airway epithelium. J. Cell Sci. 125, 932-942 (2012).

21. Frank, D. B. et al. Emergence of a wave of Wnt signaling that regulates lung alveologenesis by controlling epithelial self-renewal and differentiation. Cell Rep. 17, 2312-2325 (2016).

22. Guha, A. et al. Neuroepithelial body microenvironment is a niche for a distinct subset of Clara-like precursors in the developing airways. Proc. Natl Acad. Sci. USA 109, 12592-12597 (2012) 
23. Cai, Y. et al. Transgenically-expressed secretoglobin $3 A 2$ accelerates resolution of bleomycin-induced pulmonary fibrosis in mice. BMC Pulm. Med. 15, 72 (2015).

24. Reynolds, S. D. et al. Conditional stabilization of $\beta$-catenin expands the pool of lung stem cells. Stem Cells 26, 1337-1346 (2008).

25. Tzouvelekis, A. et al. Comparative expression profiling in pulmonary fibrosis suggests a role of hypoxia-inducible factor-1alpha in disease pathogenesis. Am. J. Respir. Crit. Care Med. 176, 1108-1119 (2007).

26. Bodempudi, V. et al. miR-210 promotes IPF fibroblast proliferation in response to hypoxia. Am. J. Physiol. Lung Cell. Mol. Physiol. 307, L283-L294 (2014).

27. Gonzalez, R. F., Allen, L., Gonzales, L., Ballard, P. L. \& Dobbs, L. G. HTII-280, a biomarker specific to the apical plasma membrane of human lung alveolar type II cells. J. Histochem. Cytochem. 58, 891-901 (2010).

28. Jarriault, S. et al. Signalling downstream of activated mammalian Notch. Nature 377, 355-358 (1995).

29. Chen, $X$. et al. XBP1 promotes triple-negative breast cancer by controlling the HIFlalpha pathway. Nature 508, 103-107 (2014).

30. Chi, J. T. et al. Gene expression programs in response to hypoxia: cell type specificity and prognostic significance in human cancers. PLoS Med. 3, e47 (2006).
31. Holland, S. J. et al. R428, a selective small molecule inhibitor of Axl kinase, blocks tumor spread and prolongs survival in models of metastatic breast cancer. Cancer Res. 70, 1544-1554 (2010)

32. Amato, K. R. et al. Genetic and pharmacologic inhibition of EPHA2 promotes apoptosis in NSCLC. J. Clin. Invest. 124, $2037-2049$ (2014).

33. Sommer, N., Strielkov, I., Pak, O. \& Weissmann, N. Oxygen sensing and signal trans duction in hypoxic pulmonary vasoconstriction. Eur. Respir. J. 47, 288-303 (2015).

34. Gustafsson, M. V. et al. Hypoxia requires notch signaling to maintain the undifferentiated cell state. Dev. Cell 9, 617-628 (2005).

35. Sahlgren, C., Gustafsson, M. V., Jin, S., Poellinger, L. \& Lendahl, U. Notch signaling mediates hypoxia-induced tumor cell migration and invasion. Proc. Natl Acad. Sci. USA 105, 6392-6397 (2008)

36. Iyer, N. V. et al. Cellular and developmental control of 02 homeostasis by hypoxiainducible factor $1 \alpha$. Genes Dev. 12, 149-162 (1998).

37. Evans, M. J., Cabral, L. J., Stephens, R. J. \& Freeman, G. Transformation of alveolar Type 2 cells to Type 1 cells following exposure to NO2. Exp. Mol. Pathol. 22 142-150 (1975).

38. Rock, J. R. et al. Basal cells as stem cells of the mouse trachea and human airway epithelium. Proc. Natl Acad. Sci. USA 106, 12771-12775 (2009). 


\section{METHODS}

Animals and treatment All animal procedures were approved by the Institutional Animal Care and Use Committee of UCSF and all animal experiments were done in compliance with ethical guidelines and the approved protocols. HIFl $\alpha^{f / f l}$ (ref. 39), Shh-Cre (ref. 40), Krt5-CreERT2 (ref. 41), Sox2-CreERT2 (ref. 42), $\beta$-catenin ${ }^{\text {loxEx }} 3$ (ref. 43), CC10-CreERT (ref. 44), FoxJ1-CreERT2 (ref. 45), p63-CreERT2 (ref. 46), Ub-GFP (ref. 47), SPC-CreERT2 (ref. 48), and Ail4-tdTomato (ref. 49) mice were previously described. For all experiments, 6-8-week-old animals of both sexes were used in equal proportions. All animal studies utilized a minimum of 3 mice per group.

For influenza infection, mice were administered $280 \mathrm{FFU}$ of Influenza H1N1 (PR8) intranasally (survival rate $>90 \%$ ). Briefly, PR8 virus dissolved in $30 \mu \mathrm{l}$ of PBS was pipetted onto the nostrils of heavily anesthetized mice (agonal breathing), whereupon mice aspirated the fluid directly into their lungs.

For all animal studies, no statistical method was used to predetermine sample size. The experiments were not randomized, and the investigators were not blinded to allocation during experiments and outcome assessment.

Lineage tracing. For analysing the lineage fate of Sox2-expressing cells, a single dose of $0.25 \mathrm{mg} \mathrm{g}^{-1}$ body weight tamoxifen dissolved in $50 \mu \mathrm{l}$ corn oil was administered i.p. one week before PR8 infection. For HIF1 $\alpha$ deletion in Sox $2^{+}$cells, three doses of tamoxifen were utilized to ensure complete deletion. To trace Krt5 ${ }^{\text {pos }}$ cells, a single dose of $0.125 \mathrm{mg} \mathrm{g}^{-1}$ tamoxifen was administered i.p. at day 7 to 10 postPR8 infection when Krt5 ${ }^{\text {pos }}$ cells were abundant. To purify LNEPs by excluding club cells and multi-ciliated cells, five doses of $0.25 \mathrm{mg} \mathrm{g}^{-1}$ body weight tamoxifen were administered to CC10-CreERT; FOXJ1-CreERT2; Ai14-tdTomato mice i.p. two weeks before euthanizing the mice to maximize the recombination. To determine the cell of origin for post-influenza Krt5 ${ }^{+}$cells, p63-CreERT2/tdTomato mice were administered five doses of $0.25 \mathrm{mg} \mathrm{g}^{-1}$ body weight tamoxifen. We waited 6 weeks for tamoxifen clearance and then infected as described above. Background (tamoxifenindependent) recombination in $\mathrm{p} 63$-CreERT2 mice is $<1 \%$.

Tissue hypoxia detection To detect hypoxic regions in tissue, mice received pimonidazole hydrochloride (60 $\mathrm{mg} \mathrm{kg}^{-1}$ body weight, i.p., Hypoxyprobe Plus kit) $1 \mathrm{~h}$ before euthanization. The lungs were inflated with $1 \%$ paraformaldehyde (PFA), incubated with $30 \%$ sucrose overnight and embedded with OCT. Sections $(80 \mu \mathrm{m}$ thick) were cut and air-dried overnight on Superfrost plus slides (Fisher Scientific). The sections were stained with FITC-conjugated anti-pimonidazole mouse IgG (1:100, 4.3.11.3 mouse FITC-MAb, Hypoxyprobe Plus kit) together with rabbit anti-Krt5 (1:1,000; Covance, no. PRB-160P) overnight in PBS plus 1\% BSA, 5\% nonimmune horse serum (UCSF Cell Culture Facility), 0.3\% Triton X-100 (Sigma), and $0.02 \%$ sodium azide (Sigma), followed by secondary antibody and DAPI staining. The slides were mounted with Prolong (Invitrogen) and imaged with a Yokogawa spinning-disk confocal (UCSF Biological Imaging Development Center).

Saturated phosphatidylcholine (SatPC) measurement SatPC was isolated from lungs of newborn animals. Lungs were first weighed, then placed in ice-cold $0.9 \%$ saline and pulse sonicated. Total lipids were extracted as previously described (ref. 50). SatPC was isolated as previously described (ref. 51) and normalized to lung weight.

Pulse oximetry. Arterial oxygen saturation was measured using the MOUSEOX Pulse Oximeter system (Starr Life Science). Mice were shaved prior to infection and the CollarClip utilized so that measurements could be taken without anesthesia. Measurements were taken for at least $3 \mathrm{~min}$ per mouse after establishing the first successful reading. Error-free measurements were then averaged for each time point for each mouse.

Excess lung water analysis. This analysis was performed as previously described ${ }^{52}$. Briefly, the lungs were removed, weighed and homogenized (after addition of $1 \mathrm{ml}$ distilled water). The blood was collected through right-ventricle puncture. The homogenate was weighed and a fraction was centrifuged $(10,000 \mathrm{~g}, 8 \mathrm{~min})$ for assay of haemoglobin concentration in the supernatant. Another fraction of homogenate, supernatant, and blood were weighed and then desiccated in an oven $\left(60^{\circ} \mathrm{C}\right.$ for $24 \mathrm{~h}$ ). Calculations of water fraction, blood volume, water volume, whole lung dry weight, and ultimately excess lung water calculations were performed using equations previously described ${ }^{52}$. The mice used for this analysis were the same cohort used for pulse oximetry.

Tissue preparation and immunofluorescence (IF) Freshly dissected mouse lungs were inflated with 4\% PFA plus 50\% OCT dissolved in PBS for $1 \mathrm{~h}$ at room temperature. After fixation, the lungs were washed with PBS and incubated with $30 \%$ sucrose plus 50\% OCT overnight, then embedded in OCT and frozen the following day. Cryosections ( $7 \mu \mathrm{m}$ thick) were cut and fixed for an additional $5 \mathrm{~min}$ in $4 \%$ PFA at room temperature, then incubated with $0.1 \%$ sodium borohydride (Sigma-Aldrich) in PBS to reduce aldehyde-induced background fluorescence for $3 \times 10$-min intervals, and subsequently blocked and stained in PBS plus 1\% BSA (Affymetrix), 5\% nonimmune horse serum, $0.1 \%$ Triton $\mathrm{X}-100$, and $0.02 \%$ sodium azide.

Cells spun on slides were fixed with $4 \%$ PFA for $5 \mathrm{~min}$ before cytospin and then stained as above. Cell grown on Matrigel were fixed in IHC Zinc Fixative (BD) for $5 \mathrm{~min}$ and subsequently blocked and stained in TBS-based blocking buffer, as the zinc fixative reacts with phosphate.

The following antibodies were used for IF: rabbit anti-pro-SPC (1:3,000; Millipore, no. AB3786), goat anti-pro-SPC (1:2,000; Santa Cruz, no. SC-7706), rabbit anti-Krt5 (1:1,000; Covance, no. PRB-160P), chicken anti-Krt5 (1:1,000; Covance, no. SIG-3475), rabbit anti- $\Delta$ Np63 (1:100; Cell Signaling, no. 13109), rat anti-mouse integrin $\beta 4$ (1:200; BD, no. 555721), goat anti-CC10 (1:10,000, a gift from B. Stripp, Cedars-Sinai Medical Center, USA), mouse anti-acetylated tubulin (1:500, Sigma, 6-11B-1), rat anti-E-cadherin (1:500, Invitrogen, no. 13-1900), rabbit anti-Hes1 (1:1,000; Cell Signaling, no. 11988), goat anti-Scgb3a2 (1:100, R\&D, AF3465).

Quantification of immunofluorescence. To quantify Krt5 $5^{\text {pos }}$ or AEC2-depleted area, mosaic images covering the whole lobes were generated from multiple $\times 10$ fields captured on a Zeiss AxioImager upright fluorescent microscope and tiled using $10 \%$ image overlap by Axiovision 4.7 software. The $\mathrm{Krt}^{\mathrm{pos}}$ or $\mathrm{SPC}^{\mathrm{pos}}$ areas and total areas were measured using outline spline in the measure menu of Axiovision 4.7. At least 3 sections, each section containing $2-3$ individual lobes and separated by over $300-\mu \mathrm{m}$ depth were quantified for each mouse ( $n \geq 5$ mice).

To lineage trace Sox2-expressing cells into alveolar cell fate, all the traced cells in injured alveolar area were captured at $\times 20$ magnification and counted using events in the measure menu of Axiovision 4.7. On average, $>3,000$ cells were counted per section. For each mouse, we quantified at least 2 sections, each containing 2-3 individual lobes and separated by over $300-\mu \mathrm{m}$ depth ( $n \geq 4$ mice).

To quantify p63-CreERT2 trace of $\mathrm{Krt}^{+}$cells post-influenza, images were captured as above and cells were counted from $>4$ sections per mouse and included at least 3 individual lobes. In total, $>3,200$ cells were scored from $n=3$ mice.

To quantify Krt5 $5^{\text {pos }}$ or $\mathrm{SPC}^{\text {pos }}$ cell percentage in cultured LNEPs accurately, the colonies were dissociated from Matrigel and digested into single-cell suspension as described below, followed by cytospin and staining. Stained cells were captured at $\times 20$ and at least 600 cells were counted per condition.

Mouse lung epithelial cell isolation and flow cytometry. Mouse lung epithelial cells were isolated as previously described ${ }^{2}$. For FACS analysis, single-cell preparations were negatively selected with biotinylated antibodies: rat anti-mouse CD45 (1:200, $\mathrm{BD}$, no. 553078), rat anti-mouse CD16/CD32 (1:200, BD, no. 553143), rat antimouse CD31 (1:200, BD, no. 553371), and then incubated for $1 \mathrm{~h}$ at $4{ }^{\circ} \mathrm{C}$ with the following primary antibodies or viability dye diluted in DMEM (without phenol red) plus 2\% FBS (Gibco): phycoerythrin (PE) or BV421-conjugated rat antimouse EpCAM (1:500; Biolegend, no. 563477, no. 563214), Alexa Fluor 647 or PEconjugated rat anti-mouse integrin $\beta 4$ (1:75; BD, no. 553745), fixable viability dye eFluor 780 (1:2,000, eBioscience). Sorting and analysis was performed on BD FACS Aria cytometers. Of note, so far we do not have a method to isolate completely pure LNEPs. For culture, we used EpCAM ${ }^{\text {pos }} \beta 4^{\text {pos }}$ cells, which have a contamination of club cells and multi-ciliated cells, but the contaminated cells do not grow in culture. To isolate highly purified LNEPs, we used CC10/FOXJ1 double Cre mice to exclude club and multi-ciliated cells, leading to similar results as the method we developed in a previous study using CD14 and CD200 antibodies ${ }^{2}$.

For intracellular flow, single-cell preparations were negatively selected and stained with eFluor 780 as above, then fixed with IHC Zinc Fixative (BD) for $30 \mathrm{~min}$ at $4^{\circ} \mathrm{C}$, permeabilized with PBS pH 7.2, $0.1 \%$ bovine serum albumin (BSA), $0.2 \%$ saponin for $5 \mathrm{~min}$, and stained with primary and secondary antibodies in permeabilization buffer sequentially. Goat anti-pro-SPC (1:500; Santa Cruz, no. SC-7706), BV421 rat anti-mouse EpCAM, and Alexa Fluor 488 donkey anti-goat $\operatorname{IgG}(1: 2,000$, ThermoFisher, no. A-11055) were used.

\section{Cell line identity. No cell lines were used in this study.}

Mouse LNEPs culture. Mouse lung LNEPs were flow sorted by $\mathrm{EpCAM}^{\mathrm{pos}} \beta 4^{\text {pos }}$ and cultured on Matrigel (BD) as follows. A 48-well plate or 8-well chamber slide was coated with $150 \mu \mathrm{l}$ Matrigel per well, allowed to solidify at $37^{\circ} \mathrm{C}$, and then equilibrated with SABM (Lonza) for at least $30 \mathrm{~min}$ prior to cell plating. A total of 15,000 to 25,000 cells were plated in each well and maintained in SAGM (Lonza) supplemented with 5\% charcoal-stripped FBS (ThermoFisher), $10 \mathrm{ng} \mathrm{ml}^{-1}$ KGF (FGF-7, Peprotech), $10 \mu \mathrm{M} \mathrm{Y-27632} \mathrm{(Sigma)} \mathrm{and} 50 \mathrm{ng} \mathrm{ml}^{-1}$ murine noggin (Peprotech) for the first 2 days, and then replaced with SAGM supplemented with $5 \%$ charcoal-stripped FBS and $10 \mathrm{ng} \mathrm{ml}^{-1}$ KGF with or without CHIR99021 (2 nM dissolved in dimethylsulfoxide (DMSO)) for an additional 5-7 days. Cells were passed once a week by incubation with $25 \mathrm{U} \mathrm{ml}^{-1}$ Dispase II (Roche) at $37^{\circ} \mathrm{C}$ for 
$20 \mathrm{~min}$ to liberate colonies and followed by $15 \mathrm{~min}$ incubation with $2 \mathrm{mM}$ EDTA in PBS at $37^{\circ} \mathrm{C}$ to dissociate the colonies and obtain a single-cell suspension. The cells were then plated on Matrigel and maintained in SAGM supplemented with 5\% charcoal-stripped FBS and $10 \mathrm{ng} \mathrm{ml}^{-1}$ KGF with or without CHIR99021 through additional passages. Cells were gradually activated (expressed Krt5) over time in culture, as described in the manuscript.

For air/liquid interface experiments, mouse LNEPs were isolated as described above and grown for a single passage in submersion conditions to establish the culture. Cells were then liberated and replated into continued Matrigel submersion culture or on Matrigel layered on top of a Transwell membrane exposed to air (COSTAR Transwell Permeable Support, no. 3413), cultured for 7 days, and then isolated as above for RNA. For hypoxyprobe staining, $2 \mu \mathrm{M}$ pimonidazole was added to culture media in each condition for $1 \mathrm{~h}$ on the seventh day after seeing. Cells were then washed thoroughly with TBS before zinc fixation and stained as described above.

For LNEP isolation from p63-CreERT2/tdTomato mice, mice were treated with 5 doses of tamoxifen, and $\mathrm{EpCAM}^{\text {pos }} \beta 4^{\text {pos }}$ cells were flow sorted and cultured as normal. Wells were visually inspected to confirm that cultures only rarely contained endogenous p63-labelled (tdTomato ${ }^{+}$) cells. Labelling was induced by a $24 \mathrm{~h}$ pulse of $100 \mathrm{nM}$ hydroxytamoxifen (4OHT) $24 \mathrm{~h}$ after re-plating for the second passage ( $\sim 10$ days after isolation), demonstrating induction of p63 expression in cells that were p63 ${ }^{\text {neg }}$ in situ (see Supplementary Fig. 4d).

Migration assay. Transwell assays were performed with Corning BioCoat Invasion Chambers (inserts coated with Matrigel, $8.0 \mu \mathrm{m}$ pore size). Primary human basalenriched and AEC2s (80,000 and 200,000 respectively) were seeded on top of the inserts in SAGM with KGF, Noggin and Y-27632 for 2-3 days for recovery, then washed with PBS and starved with SABM for $6 \mathrm{~h}$ before migration through Matrigel toward $1 \% \mathrm{FBS}$ plus $50 \mathrm{ng} \mathrm{ml}^{-1}$ EGF in SAGM at $37^{\circ} \mathrm{C}$ for $\sim 66 \mathrm{~h}$ (with SABM on top of the inserts). Non-invaded cells and Matrigel were removed by swabbing. Invaded cells were fixed in methanol, stained with $1 \%$ crystal violet (Sigma-Aldrich), and imaged with a Zeiss AxioImager at $\times 10$ magnification. Invaded cells were counted in random fields avoiding the edge of the insert.

Wound closure assays were performed using specific culture inserts (Ibidi, no. 80209). A culture insert was transferred to an individual well of a 24 -well plate and the surface in the insert was coated with 1:20 diluted Matrigel for $2 \mathrm{~h}$ before cell seeding. Activated LNEPs were disaggregated as described above and resuspended in SAGM with KGF, Noggin and Y-27632 at a density of 0.7-1 million $\mathrm{ml}^{-1}$. Seventy microlitres of cell suspension was seeded into each well of the insert. After cell attachment for about $24 \mathrm{~h}$ forming a confluent layer, the cells were pretreated with inhibitors for $1 \mathrm{~h}$ before removing the culture inserts, and then washed with PBS twice and incubated with fresh SAGM medium in the presence/absence of the AXL inhibitor R428 (Apexbio) or the EPHA2 inhibitor ALW-II-41-27 (Apexbio) dissolved in DMSO. The cell migration into the defined cell-free gap $(500 \mu \mathrm{m})$ was observed for $24 \mathrm{~h}$ under an inverted microscope (Nikon). Images of the cell-free gap were captured at $0 \mathrm{~h}$ and $24 \mathrm{~h}$, and total cell-free area was quantified with Axiovision 4.7.

Chromatin immunoprecipitation (ChIP). ChIP experiments were performed following the Imprint Chromatin Immuno-precipitation protocol (Sigma). Expanded LNEP colonies were digested as described above, seeded on 1:20 diluted Matrigel-coated $10 \mathrm{~cm}$ dishes and harvested on reaching $80-90 \%$ confluence. Cells were crosslinked with $4 \%$ formaldehyde for $10 \mathrm{~min}$ followed by nuclei isolation, sonication to shear DNA into $100-500 \mathrm{bp}$ fragments, protein/DNA complex pulldown and crosslink reversal. One microgram of rabbit anti-Cleaved Notch1 (Cell Signaling, no. 4147) and goat anti-HIF1 $\alpha$ (R\&D Systems, no. AF1935) antibody were used for each IP. The precipitated DNA and input DNA was quantified by qPCR using specific primers on Krt5, Heyl and Hes5 promoters.

Human tissues. All human normal and fibrotic tissue samples were obtained from UCSF Interstitial Lung Disease Blood and Tissue Repository and are classified as Non-identifiable Otherwise Discarded Human Tissues, for which no consent/IRB approval is required. Slides from lung tissue blocks of non-identified human subjects with documented H1N1 influenza A infection 10-21 days after diagnosis and without secondary bacterial infection, as judged by PCR screening, were obtained from A. Denison at the Center for Disease Control (Atlanta).

Human lung epithelial cell isolation. Distal lung tissue was obtained and dissected into roughly $5 \mathrm{~cm}^{3}$ pieces. Tissue was washed in $500 \mathrm{ml}$ sterile PBS for $10 \mathrm{~min}$ at $4{ }^{\circ} \mathrm{C}$ at least two times, or until PBS no longer appeared obviously bloody. An additional 10 min wash was then performed with Hank's buffered saline solution (HBSS). Using autoclaved Kim Wipes, tissue was compressed to remove as much liquid as possible and further dissected into $1 \mathrm{~cm}^{3}$ pieces. Sterile HBSS buffer containing $5 \mathrm{U} \mathrm{ml}^{-1}$

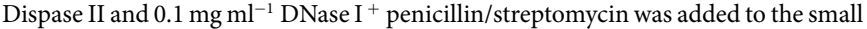
tissue pieces. Tissue rapidly takes up the digest solution at this point, becoming visibly engorged. Tissue was digested for $2 \mathrm{~h}$ at $37^{\circ} \mathrm{C}$ and Fungizone (1:400) was added for the final $30 \mathrm{~min}$ of the digest. The digest solution was then stored overnight at $4{ }^{\circ} \mathrm{C}$ without further degradation of cells due to lack of Dispase activity at this temperature. The following day, the tissue in digestion solution was warmed to $37^{\circ} \mathrm{C}$ and liquefied using an Osterizer 12 Speed Blender as follows: $5 \mathrm{~s}$ pulse, $5 \mathrm{~s}$ grate, and $2-5 \mathrm{~s}$ pulse. The suspension was poured through a glass funnel lined with sterile $4 \times 4$ gauze, applying some compression to recover as much of the solution as possible. The cell suspension was sequentially filtered through $100 \mu \mathrm{m}, 70 \mu \mathrm{m}$ and $40 \mu \mathrm{m}$ strainers. Finally, red blood cells were removed using Red Blood Cell Lysis Buffer (Sigma-Aldrich).

Single-cell RNA-Seq of human lungs. The cell preparations were stained as described for mouse cells with corresponding anti-human antibodies: HTII-280 (1:100, a generous gift from L. Dobbs, University of California - San Francisco, USA), anti-human/mouse CD49f (1:100, Biolegend, no. 313602), PE anti-human EpCAM (1:400, Biolegend, no. 324206), and anti-human CD45 APC-Cy7 (1:200, Biolegend, no. 304014). As judged by cytospin, all the SPC ${ }^{\text {pos }}$ cells were captured by HTII-280 sort. Sorted single cells were captured on a small-sized $(7-10 \mu \mathrm{m}$ cell diameter) microfluidic mRNA seq chip (Fluidigm) using the Fluidigm C1 Single-Cell Auto Prep System. The downstream steps (lysis, cDNA synthesis/amplification, library preparation) were carried out following the Fluidigm $\mathrm{C} 1$ protocol, incorporating the Illumina Nextera XT DNA Sample Preparation Kit. Nextera libraries consisting of barcoded single-cell samples were pooled and sequenced on an Illumina HiSeq 2,500 sequencer using high-output mode with $100 \mathrm{bp}$ paired-end reads. This results in a sequencing depth of $\sim 2-3$ million reads per single-cell sample. Pairedend fastq files were aligned using the Tophat/Bowtie2 software $^{53}$ and annotated using the Cufflinks package ${ }^{54}$ and the UCSC hg19 index (Illumina), followed by normalization using Cuffnorm. Cells with a fragment mapping rate below $50 \%$ and less than 1,000 genes sequenced (1 FPKM threshold) were filtered out. Genes that were not expressed at 1 FPKM in at least 3 cells were also removed prior to subsequent Fluidigm Singular analysis running in $\mathrm{R}$, leading to $\sim 13,000$ genes. We successfully sequenced a total of 72 AEC2s and 23 basal-enriched cells from a normal lung, 49 AEC2s from scleroderma, 48 AEC2s from dyskeratosis congenita, and 27 AEC2s from IPF lung. Differential expression analysis from Singular is included in Supplementary Tables 1,3-6. Ingenuity pathway analysis (IPA, QIAGEN, www.qiagen.com/ingenuity) was performed on this differential expression data and is found in Supplementary Tables 2, 7-9.

RNA-Seq of mouse normal and activated LNEPs. Highly purified quiescent LNEPs $\left(\right.$ EpCAM ${ }^{\text {pos }} \beta 4^{\text {pos }} \mathrm{CC} 10^{\text {neg }}$ FoxJ $1^{\text {neg }}$ ) and activated LNEPs (Krt5-CreERT2 traced cells 17 days post infection) were flow sorted and RNA extracted using ReliaPrep RNA Tissue Miniprep kit (Promega). cDNA synthesis/amplification, library preparation and sequencing followed the same protocol used in Single Cell RNA-Seq.

Western blot analysis. Snap-frozen mouse lungs were ground into tissue powder and then lysed in the RIPA buffer (50 mM Tris- $\mathrm{HCl}, \mathrm{pH} 7.5,150 \mathrm{mM} \mathrm{NaCl}, 1 \%$ deoxycholate, $0.1 \%$ SDS, $1 \%$ Triton X-100) supplemented with Protease Inhibitor Cocktail, $1 \mathrm{mM}$ phenylmethyl sulfonyl fluoride, $1 \mathrm{mM}$ sodium vanadate, $10 \mathrm{mM}$ sodium fluoride, and Phosphatase Inhibitor Cocktail. The lysates were quantified using Pierce BCA protein assay kit (Thermo, no. 23225), normalized and blotted for HIFl $\alpha$ (1:500, R\&D Systems, no. AF1935), pro-SPC (1:500, Millipore, no. AB3786), Krt5 (1:1,000, Covance, no. PRB-160P), Cleaved Notch1 (1:1,000, Cell Signaling, no. 4147$)$, E-cadherin (1:2,000, BD, no. 610181), $\beta$-actin (1:10,000, Sigma-Aldrich, no. A5441). To detect influenza A virus, unboiled lysates in $1 \mathrm{mM}$ dithiothreitol and $2 \%$ SDS were blotted with anti-Influenza A antibody (1:1,000, Millipore, no. $\mathrm{AB} 1074)$.

Bronchoalveolar lavage. After the trachea was exposed, a 20-G catheter was inserted into the trachea for lavage. Cold PBS $(1 \mathrm{ml})$ was instilled into the mouse lungs followed by gentle aspiration repeated three times. All of the bronchoalveolar lavage fluid was centrifuged and the supernatant was collected to measure total protein content using BCA assay. The cell pellet was resuspended in Red Blood Lysis Buffer and counted using a haemocytometer.

Colony-formation assay. Ten thousand freshly sorted or cultured single LNEPs were plated onto a Matrigel-coated 8-well chamber slide and cultured for one week. Colonies were fixed with IHC Zinc Fixative and stained. Only colonies containing more than 5 nuclei were counted.

qRT-PCR analysis. Snap-frozen lungs were ground into tissue powder and RNA was isolated using the ReliaPrep RNA Tissue Miniprep kit (Promega). ReliaPrep RNA Cell Miniprep kit (Promega) was used to isolate RNA from freshly sorted cells or cultured cells. To isolate RNA from cultured LNEPs on Matrigel, colonies were dissociated from Matrigel using Dispase II first, and then the cell pellets were lysed following the Promega protocol. cDNA was synthesized using Superscript III (Invitrogen) 
and assayed for gene expression using Faststart Universal SYBR green Master Mix (Roche). PCR reaction and analysis was run on Eppendorf Mastercycler ep realplex 2. HPRT, L19 and GAPDH were used as internal controls and all of the data were normalized by L19. All primer sequences are as listed in Supplementary Table 10.

Statistics and reproducibility. For single-cell RNA-Seq analysis, Fluidigm Singular software was used. All other statistical calculations were performed using Graphpad Prism. Variance for all group data is expressed as \pm s.e.m. The statistical test used to determine significance for each experiment is stated in the corresponding figure legend. A $P$ value less than 0.05 was accepted as significant. The investigators were not blinded to allocation during experiments and outcome assessment. All images are representative of at least three independent experiments or mice of the same genotype. Western blots are representative of multiple independent experiments, and unprocessed original scans of blots are displayed in Supplementary Fig. 8. No statistical method was used to predetermine sample size and experiments were not randomized. All experiments presented were reproducible and $n$ for each experiment is presented in each figure legend.

Data availability. RNA-Seq data that support the findings of this study have been deposited in the Gene Expression Omnibus (GEO) under accession codes GSE83467 and GSE83501. Source data for the main and supplementary figures have been provided as Supplementary Table 11. All other data supporting the findings of this study are available from the corresponding author on reasonable request.

39. Ryan, H. E. et al. Hypoxia-inducible factor-1alpha is a positive factor in solid tumor growth. Cancer Res. 60, 4010-4015 (2000).

40. Harfe, B. D. et al. Evidence for an expansion-based temporal Shh gradient in specifying vertebrate digit identities. Cell 118, 517-528 (2004).

41. Van Keymeulen, A. et al. Distinct stem cells contribute to mammary gland development and maintenance. Nature 479, 189-193 (2011).
42. Arnold, K. et al. Sox2(+) adult stem and progenitor cells are important for tissue regeneration and survival of mice. Cell Stem Cell 9, 317-329 (2011).

43. Harada, N. et al. Intestinal polyposis in mice with a dominant stable mutation of the $\beta$-catenin gene. EMBO J. 18, 5931-5942 (1999).

44. Rawlins, E. L. et al. The role of Scgb1a1+ Clara cells in the long-term maintenance and repair of lung airway, but not alveolar, epithelium. Cell Stem Cell 4, 525-534 (2009).

45. Rawlins, E. L., Ostrowski, L. E., Randell, S. H. \& Hogan, B. L. Lung development and repair: contribution of the ciliated lineage. Proc. Natl Acad. Sci. USA 104, 410-417 (2007).

46. Lee, D.-K., Liu, Y., Liao, L., Wang, F. \& Xu, J. The prostate basal cell (BC) heterogeneity and the p63-positive BC differentiation spectrum in mice. Int. J. Biol. Sci. 10, 1007-1017 (2014).

47. Schaefer, B. C., Schaefer, M. L., Kappler, J. W., Marrack, P. \& Kedl, R. M. Observation of antigen-dependent CD8+ T-cell/ dendritic cell interactions in vivo. Cell. Immunol. 214, 110-122 (2001).

48. Chapman, H. A. et al. Integrin $\alpha 6 \beta 4$ identifies an adult distal lung epithelial population with regenerative potential in mice. J. Clin. Invest. 121, 2855-2862 (2011).

49. Madisen, L. et al. A robust and high-throughput Cre reporting and characterization system for the whole mouse brain. Nat. Neurosci. 13, 133-140 (2010).

50. Bligh, E. G. \& Dyer, W. J. A rapid method of total lipid extraction and purification. Can. J. Biochem. Physiol. 37, 911-917 (1959).

51. Mason, R. J., Nellenbogen, J. \& Clements, J. A. Isolation of disaturated phosphatidylcholine with osmium tetroxide. J. Lipid Res. 17, 281-284 (1976).

52. Su, X. et al. Activation of the $\alpha 7 \mathrm{nAChR}$ reduces acid-induced acute lung injury in mice and rats. Am. J. Respir. Cell Mol. Biol. 37, 186-192 (2007).

53. Langmead, B., Trapnell, C., Pop, M. \& Salzberg, S. L. Ultrafast and memory-efficient alignment of short DNA sequences to the human genome. Genome Biol. 10, R25 (2009).

54. Trapnell, C. et al. Transcript assembly and quantification by RNA-Seq reveals unannotated transcripts and isoform switching during cell differentiation. Nat. Biotechnol. 28, 511-515 (2010). 
a

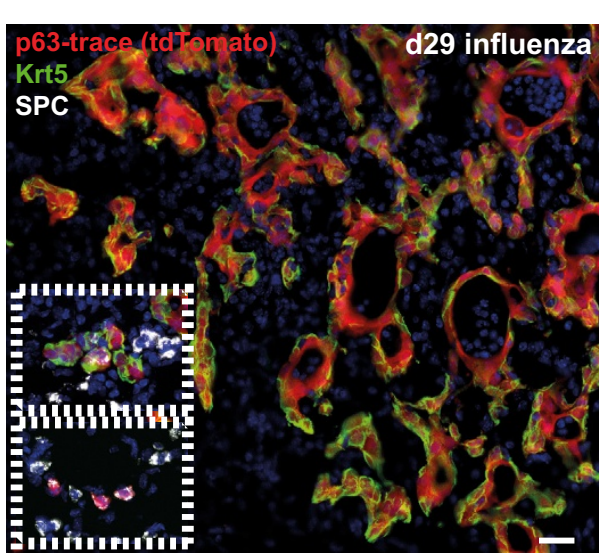

\section{b}

\section{Uninjured mouse lung \\ p63-trace (tdTomato)}

C

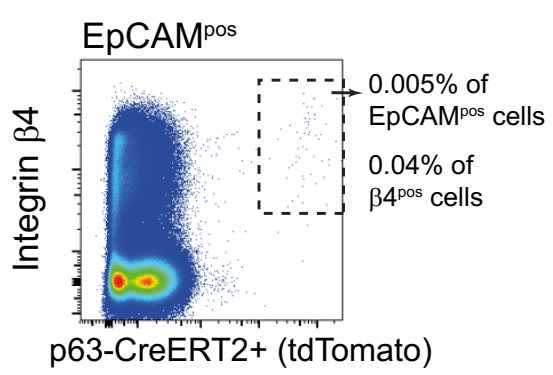

f

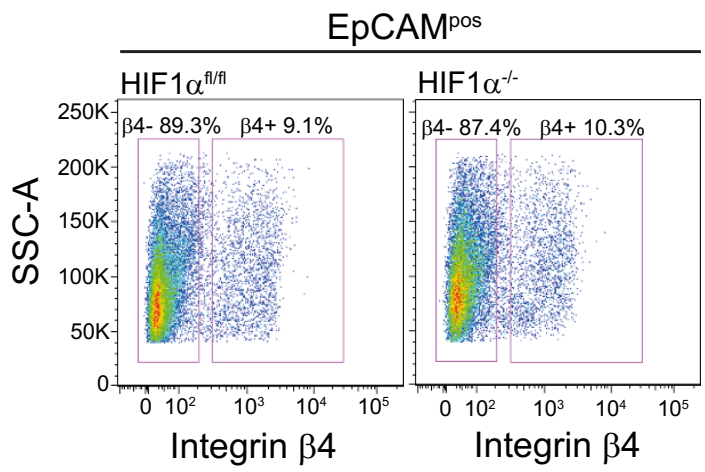

Supplementary Figure 1 p63pos LNEPs are very rare and do not efficiently generate AEC2s. Lung epithelial ablation of HIF1 a with Shh-Cre causes no defects in uninjured mice. (a) While p63 ${ }^{\text {pos }}$ LNEPs generate Krt5 metaplasia, they make only a minor contribution to AEC2 regeneration after influenza injury (insets). (b) Rare p63-CreERT2 traced (tdTomato+) cells are scattered throughout airways in uninjured mice, representing $0.005 \%$ of the total epithelium as judged by FACS analysis (c). Data represent $n=3$ mice for histology (b), $n=2$ independent experiments with pooled live cells from 3 mice for FACS (c). (d) HIFla is deleted in all the epithelial cells sorted from Shh-Cre; HIFla ${ }^{\mathrm{fl} / \mathrm{fl}}$ mouse lungs. Data are mean \pm SEM from $\mathrm{n}=3$ independent experiments, in which each group is a pool of 3 mice. (e) Lung SatPC content and (f) LNEP-enriched population (integrin $\beta 4^{\text {pos }}$ EpCAMpos) remain unchanged after HIF1 $\alpha$ deletion in epithelial cells. (e) Data are represented as mean \pm SEM from $n=4$ mice per group from two independent experiments. ( $f$ ) Data are represented as a percentage in EpCAMpos live cells from a pool of 3 mice in each group. $p$ values derived by unpaired two-tailed Student's t test. 
a

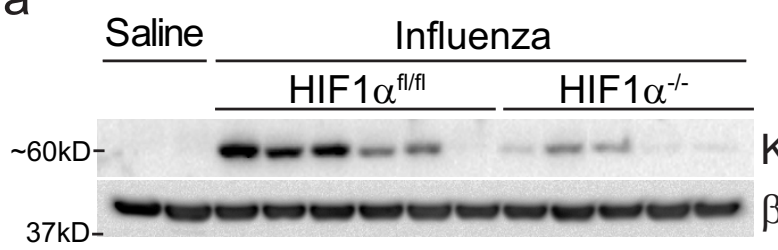

C

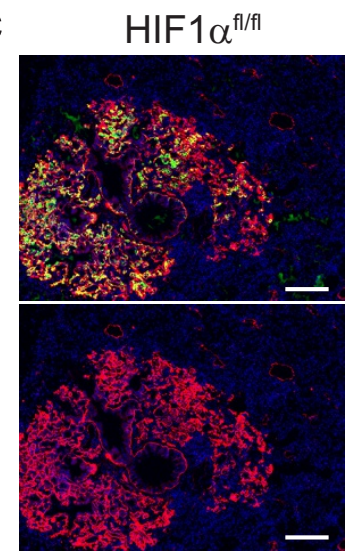

e

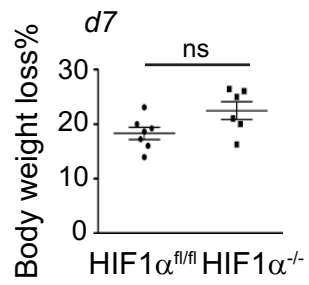

f

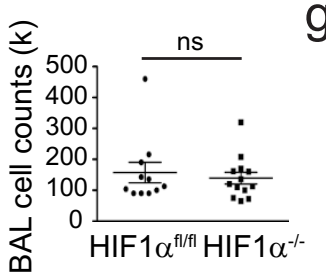

j

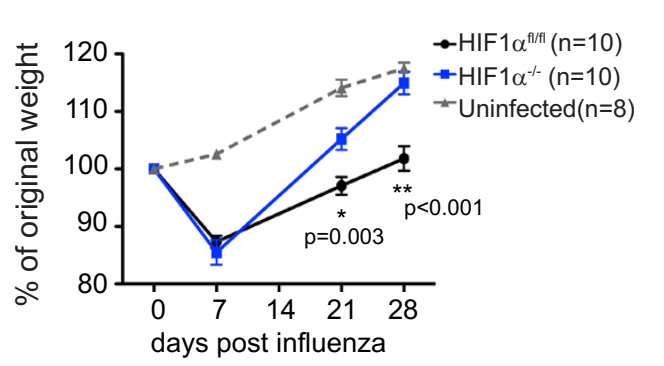

Supplementary Figure $2 \mathrm{HIF} 1 \alpha^{-/-}$mice are injured at similar levels as wildtype mice, but lack alveolar Krt5 ${ }^{\text {pos }}$ cell expansion. (a) Representative blot showing Krt5 induction is inhibited by epithelial HIF1a deletion. (b-c) No large expansion of DNp63 (b) or integrin b4 (c) positive cells in the alveoli of $\mathrm{HIFl} \alpha^{-/-}$mice, indicating alveolar Krt5 ${ }^{\text {pos }}$ cell expansion is inhibited by HIFla deletion. No difference in virus infection (d), weight loss (e), immune cell numbers in BAL $(\mathrm{f})$ and BALF protein level $(\mathrm{g})$ between wild-type and HIF1 $\alpha$ /- mice. (e-g) Data are represented as mean \pm SEM, (e) $n=7$ wild-type, $n=6$ HIFl $\alpha^{-/-}$; (f) $n=11$ wild-type, $n=13 \mathrm{HIFl}^{-/-}$; (g) n=17 wild-type, $\mathrm{n}=18 \mathrm{HIFl}^{-}$ /- mice from three independent experiments. Each data point represents one mouse. (h) Large areas of AEC2 depletion are present in both wild-type and $\mathrm{HIF} 1 \alpha^{-/-}$lungs, quantified in (i). SPC staining in airways (h, right) is an artifact b
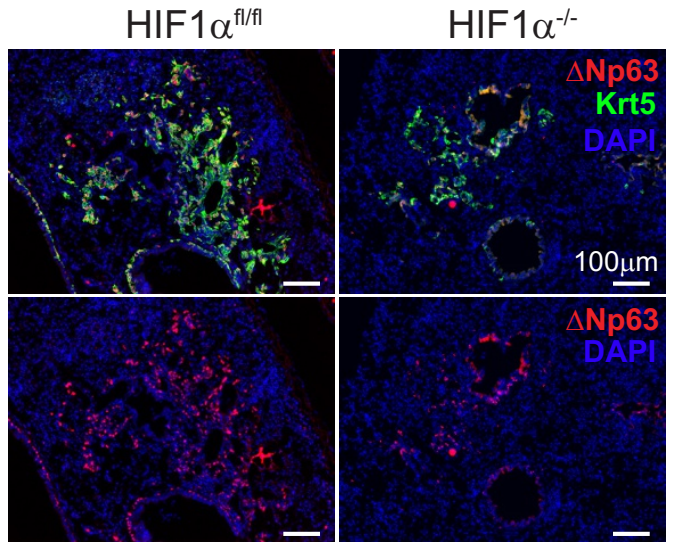

d

$50 \mathrm{kD}-$
Saline
Influenza d4
Influenza d11
$\underline{\mathrm{HIF} 1 \alpha^{\mathrm{f} / f / 1}} \underline{\mathrm{HIF} 1 \alpha^{-1-}} \underline{\mathrm{HIF} 1 \alpha^{\mathrm{f} / / \mathrm{fl}}} \underline{\mathrm{HIF} 1 \alpha^{-/-}}$

Influenza A

$37 \mathrm{kD}-$

$\beta$-actin

$\mathrm{h}$

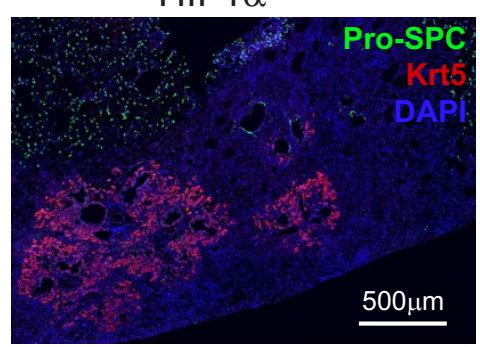

$\mathrm{HIF} 1 \alpha^{-1-}$
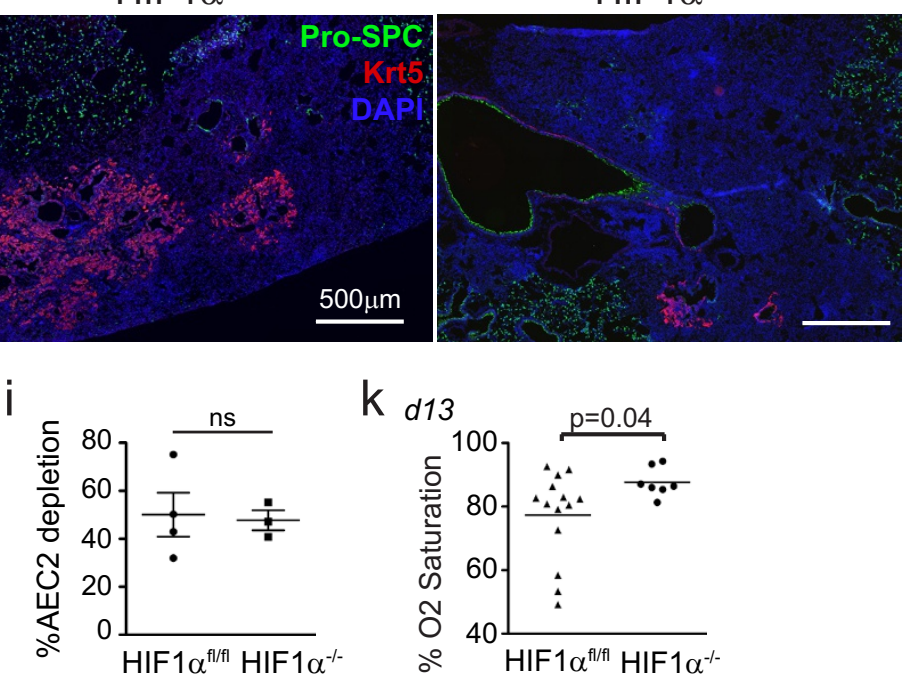

K $d 13$

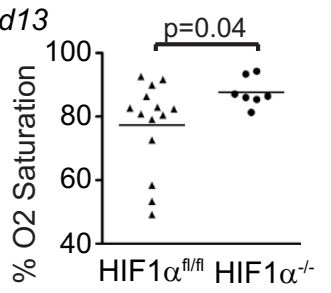

of the goat anti-pro-SPC antibody. (i) Data are represented as mean \pm SEM, $\mathrm{n}=4$ wild-type, $\mathrm{n}=3 \mathrm{HIF} 1 \alpha^{-/-}$mice from 2 independent experiments. (j) HIF1 $\alpha$ 1- mice recover weight more rapidly than their wild-type counterparts after influenza injury. Data are represented as mean $\pm S E M, n=10$ wild-type, $n=10$ $\mathrm{HIF} 1 \alpha^{-/-}, \mathrm{n}=8$ uninfected wild-type mice from three independent experiments (k) Significant difference in average arterial oxygen saturation at 13 days postinfection between $\mathrm{HIFl} \alpha^{-/-}$and wild-type mice. Each data point represents the average \% 02 saturation reading for a single mouse at this time point (see Fig. 1h). Data are mean \pm SEM, $n=7 \mathrm{HIFl}^{-/}, \mathrm{n}=14$ wild-type (2 Shh$\mathrm{Cre}^{\text {neg, }} 12$ C57BL6) mice from two independent experiments. Analysis is 11 days post-infection unless otherwise indicated. $p$ values derived by unpaired two-tailed Student's t test, except in (k) derived by Mann Whitney. 
a

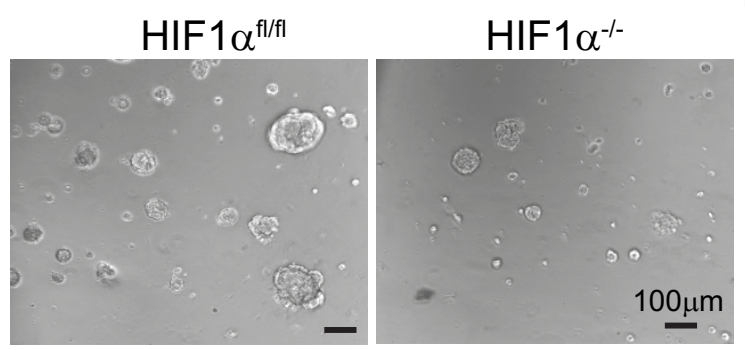

d
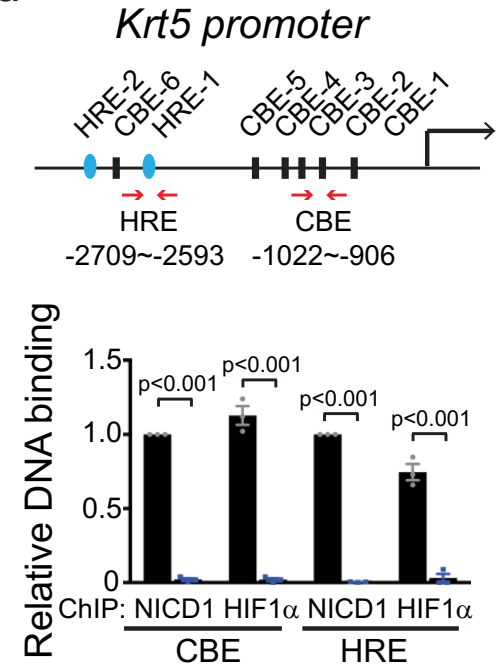

e

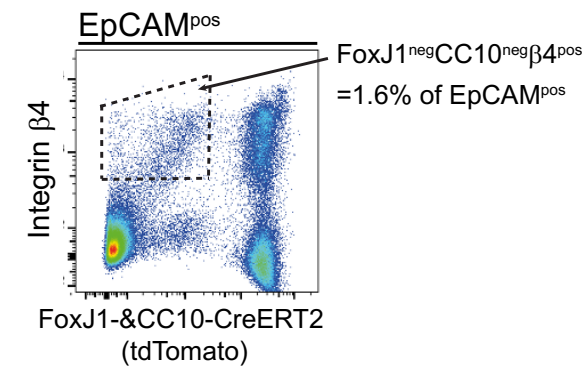

f

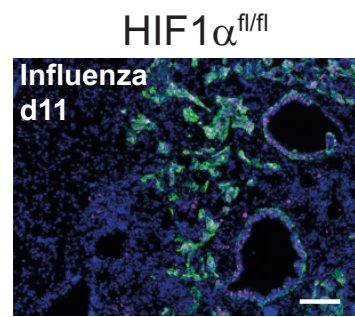

$\mathrm{HIF} 1 \alpha^{-1-}$

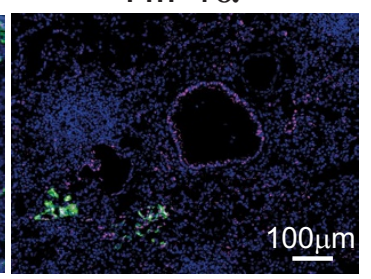

Hey1 promoter

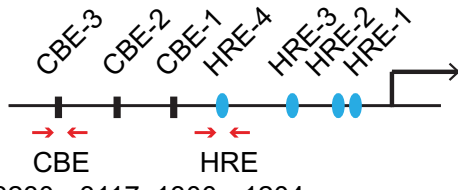

$-3230 \sim-3117-1300 \sim-1204$

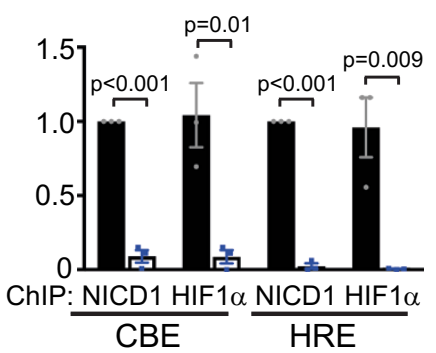

9
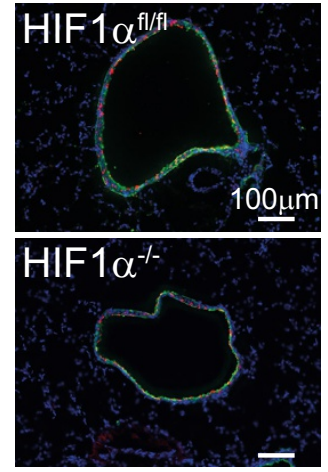

CC10/ a
C

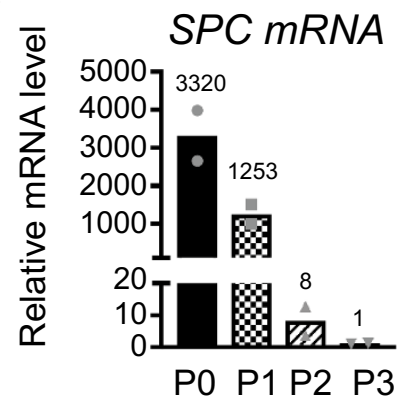

Hes5 promoter

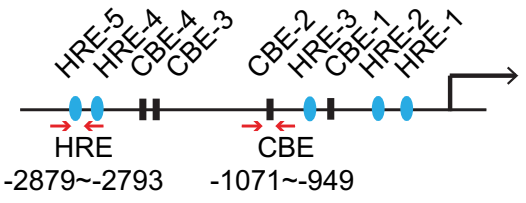

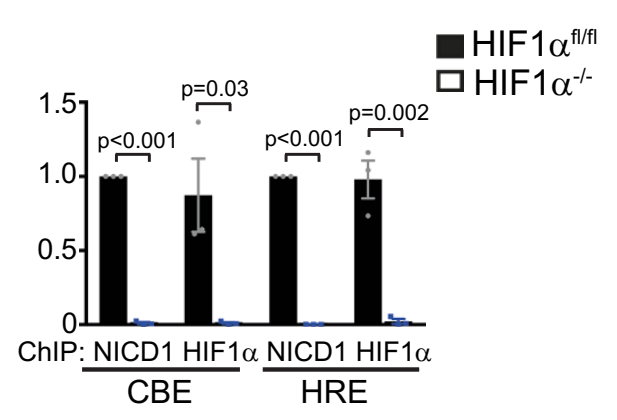

Hes1/ Krt5/ DAPI

Supplementary Figure 3 HIFl $\alpha$ promotes Notch activity in LNEPs but has no effect on airway Notch activity. (a-b) Reduced colony size and number of HIF $1 \alpha^{-1-}$ LNEPs in culture. WT, HIF $1 a^{\text {fl/fl. }}$. (c) qPCR analysis of SPC in freshly sorted (PO) and cultured (P1-P3) LNEPs showing SPC mRNA dramatically decreases upon culture, $n=2$ independent experiments. (d) Top, mouse Krt5, Hey 1 and Hes 5 promoters contain HRE and CBE. The primers used in bottom are highlighted in red. Bottom, GPCR analysis of ChIP demonstrating HIFla deletion blocks NICD1 DNA binding on Krt5, Hey1 and Hes5 promoters in cultured LNEPs. Ct value of pulled down DNA was normalized by Ct of input DNA and the abundance was calculated relative to NICD1 association of each site. (b,d) Data are represented as mean \pm SEM from $n=3$ independent experiments. $p$ values derived by unpaired twotailed Student's t test. (e) FACS isolation of highly purified LNEPS (FoxJ1 ${ }^{\text {neg }}$ $\mathrm{CC} 10^{\text {neg }}$ integrin $\beta 4+$ ) from uninjured mice used for RNA-Seq analysis. (f) HIFl 1 deletion inhibits Hes 1 staining in the alveoli but not airways. (g) HIF 1 a deletion has no effect on airway Notch activity in uninfected mice, as judged by the ratio between club cells (CC10 ${ }^{\mathrm{pos}}$ ) and multi-ciliated cells (acetylated-Tubulin ${ }^{\mathrm{pos}}$ ) remaining unchanged. 
a

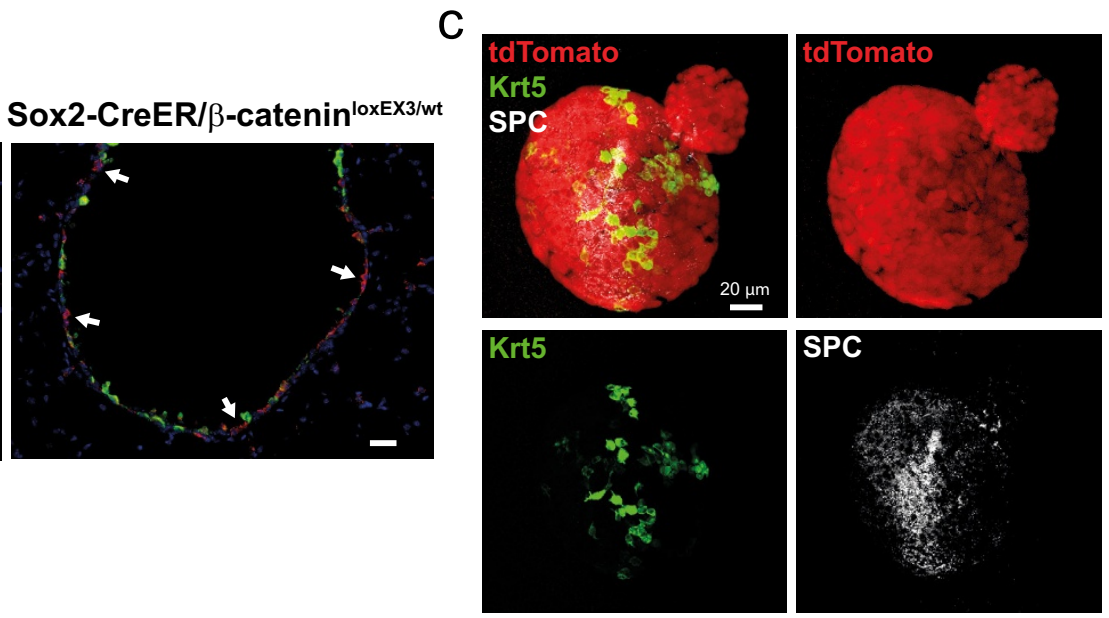

b

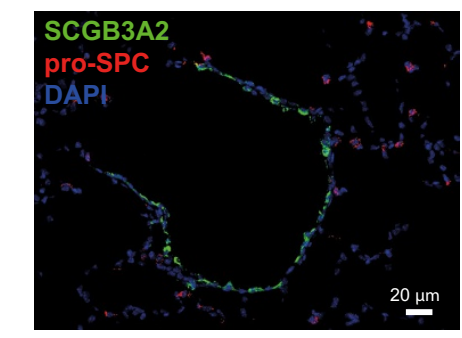

\section{C57BL6}

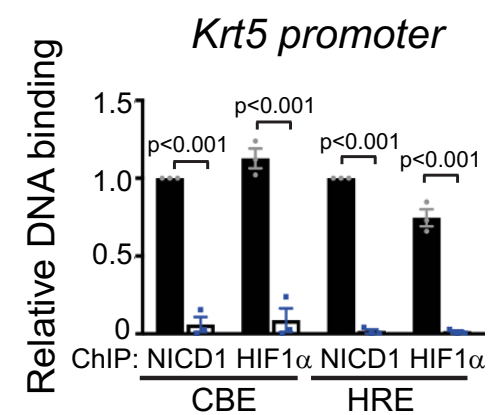

d

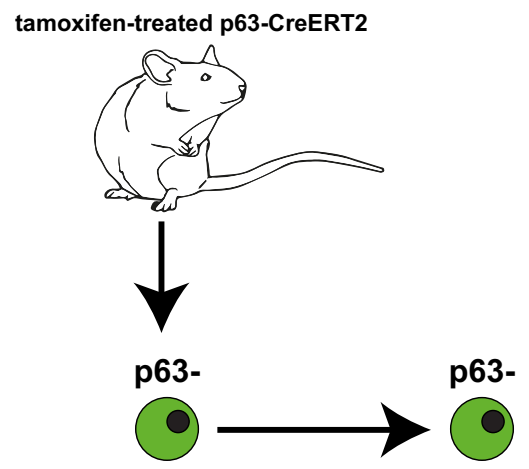

Hey1 promoter

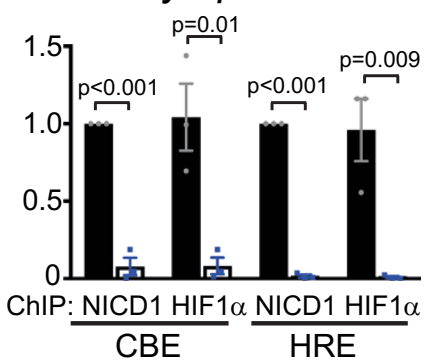

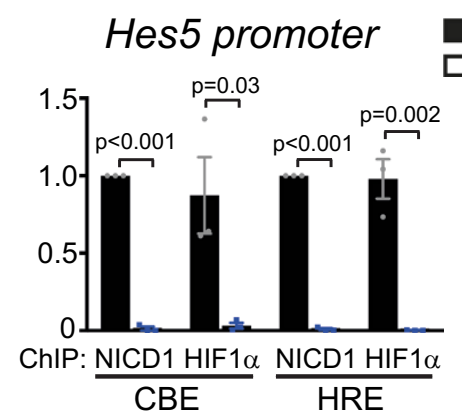

Ctl 口CHIR

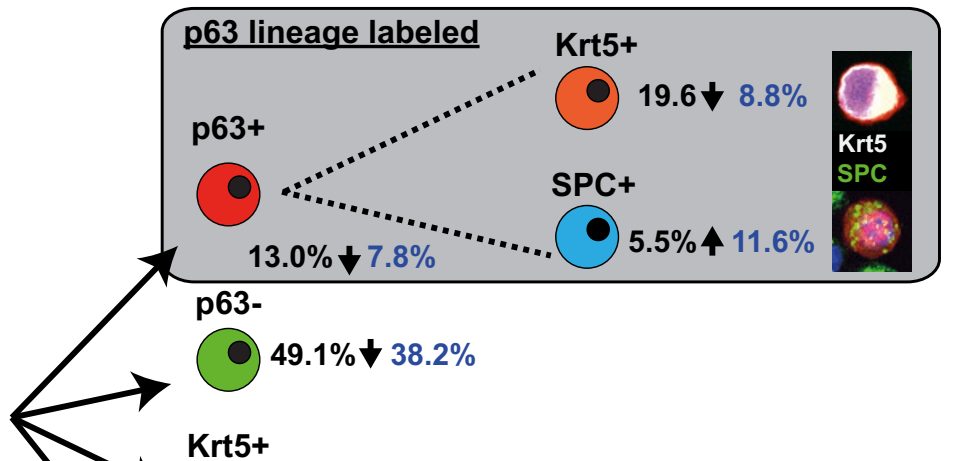

Krt5+

$6.8 \% \downarrow 3.6 \%$

SPC+

$31.1 \% 450.4 \%$

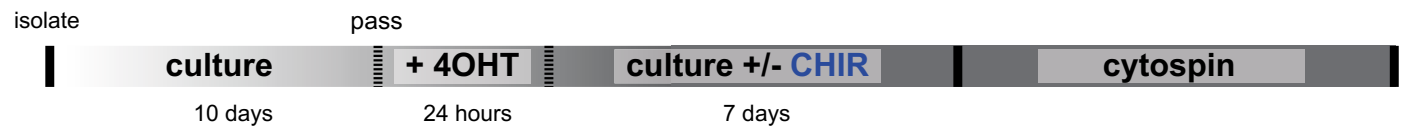

Supplementary Figure 4 Stabilization of b-catenin inhibits Notch and HIF $1 \alpha$ activity by blocking their DNA association. (a) $\beta$-catenin stabilization increases ectopic SPC expression in the airways largely independent of club cells expressing Scgb3a2. About 27\% (97 cells out of 362) Sox2traced airway cells express SPC 7 days after tamoxifen induced $\beta$-catenin stabilization, $n=3$ mice examined. (b) qPCR analysis of ChIP demonstrating NICD1 and HIFla DNA binding on Krt5, Hey1 and Hes5 promoters are blocked by CHIR. The same control sample (LNEPs from HIFla $a^{\mathrm{fl} / f l}$ mice) was used as Supplementary Fig. 3d. Data are represented as mean \pm SEM from $n=3$ independent experiments. $p$ values derived by unpaired two-tailed Student's t test. (c) Individual fluorescent channels of the colony from Fig. $3 g$ demonstrating Krt5 and SPC expression in a single clone. (d) p63 neg LNEPs in vitro either remain undifferentiated, are activated into p63 ${ }^{\text {pos }}$ cells (visualized by tdTomato expression after a brief $40 \mathrm{HT}$ treatment), or differentiate into Krt5+ or SPC+ cells. Wnt agonism (blue) results in more SPC+ cells and fewer Krt5+ cells as described in Fig. 4d. Grey inset quantifies these outcomes specifically within those cells that become p63 traced. Quantification is via immunostaining of cytospins, $n=2$ experiments. 
a
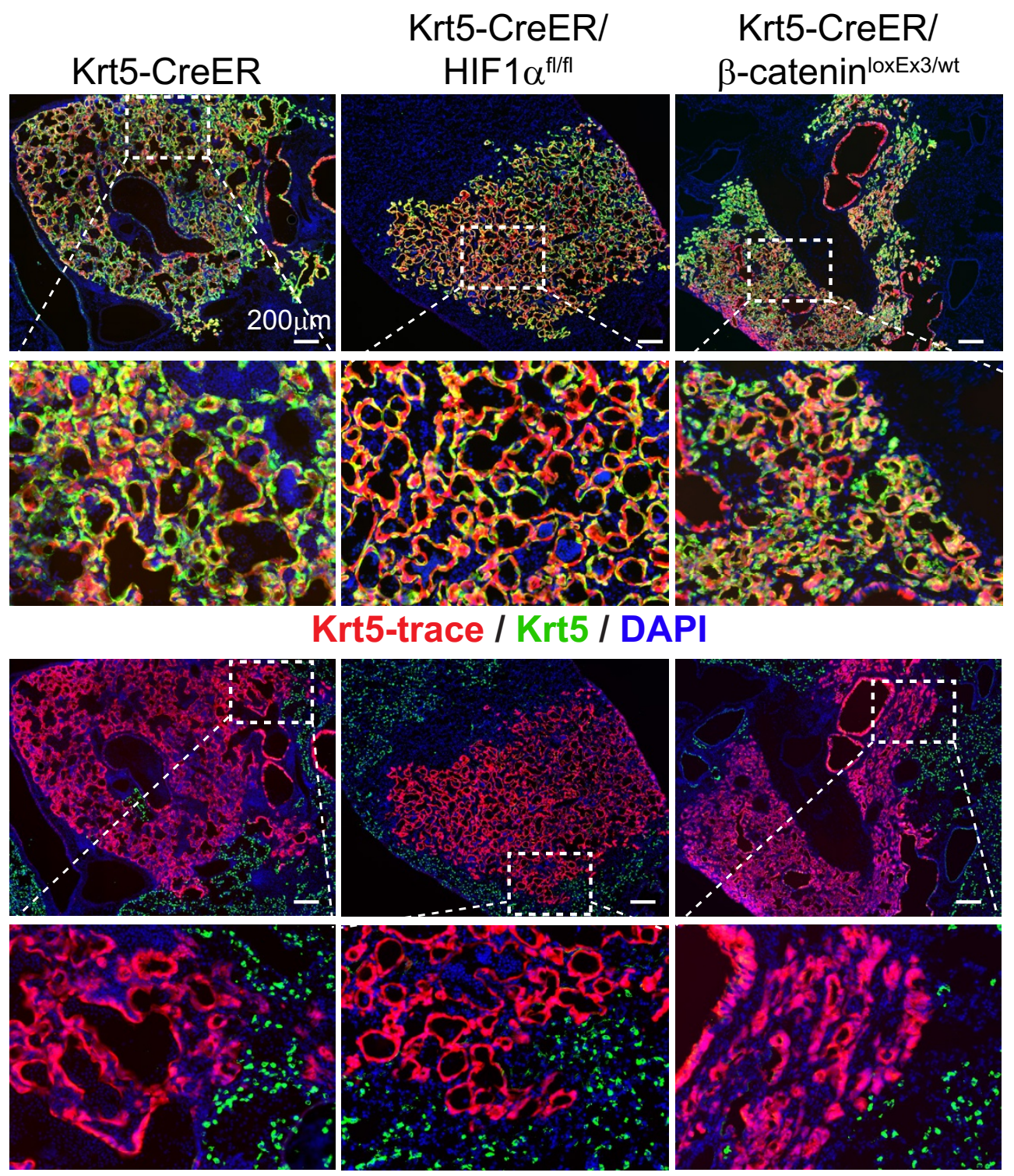

Krt5-trace / Pro-SPC / DAPI

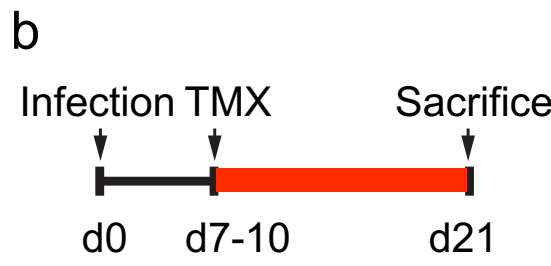

C Relative mRNA level

d Relative mRNA level

\begin{tabular}{|r|r|r|}
\hline & Krt5-trace & $\begin{array}{r}\text { Krt5-trace; } \\
\text { HIF1 } \alpha^{\mathrm{flfl}}\end{array}$ \\
\hline Hey1 & 1.0 & 1.9 \\
\hline Hey2 & 1.0 & 1.2 \\
\hline Hes5 & 1.0 & 4.0 \\
\hline HIF1 $\alpha$ & 1.0 & 0.1 \\
\hline
\end{tabular}

\begin{tabular}{|r|r|r|}
\hline & Krt5-trace & $\begin{array}{c}\text { Krt5-trace; } \\
\text { loxEx3 }\end{array}$ \\
\hline Hey1 & 1.0 & 0.9 \\
\hline Hey2 & 1.0 & 0.4 \\
\hline Hes5 & 1.0 & 0.9 \\
\hline Axin2 & 1.0 & 32280.3 \\
\hline
\end{tabular}

Supplementary Figure 5 Deleting HIFla or stabilizing b-catenin does not alter LNEP differentiation after full Notch/Krt5 activation. (a) HIFla deletion or $\beta$-catenin stabilization subsequent to Krt5 activation as described in (b) has no effect on Krt5 (green, upper panel) and SPC (green, lower panel) expression. (c) Relative mRNA levels in sorted Krt5CreERT2-traced cells 21 days post infection with $(n=8)$ or without $(n=2)$
HIF $1 \alpha$ deletion. Notch activity is not downregulated by HIF $1 \alpha$ deletion in Krt5 expressing cells. (d) Relative mRNA levels in sorted Krt5 traced cells 21 days post infection with $(n=2)$ or without $(n=5)$ b-catenin stabilization. Notch activity is not inhibited by Wnt signaling in Krt5 expressing cells. (c-d) Sorted cells from two independent experiments were pooled together for RNA isolation. 


\section{a}

Normal Human Lung Sort
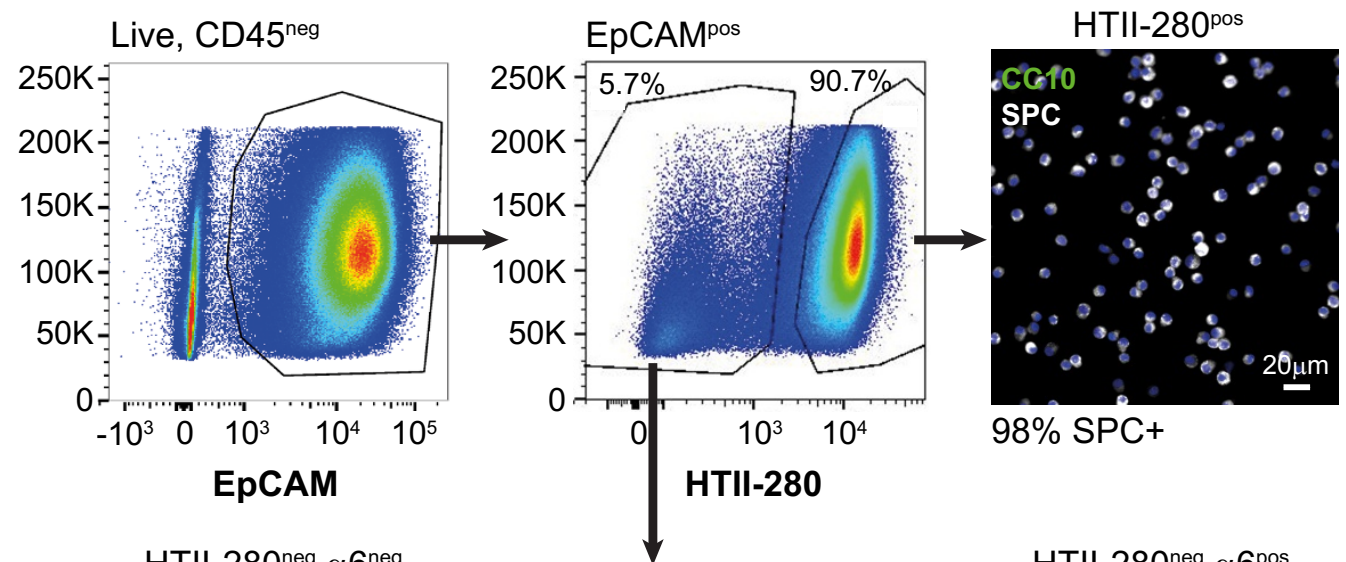

HTII-280 neg $\alpha 6^{\text {neg }}$
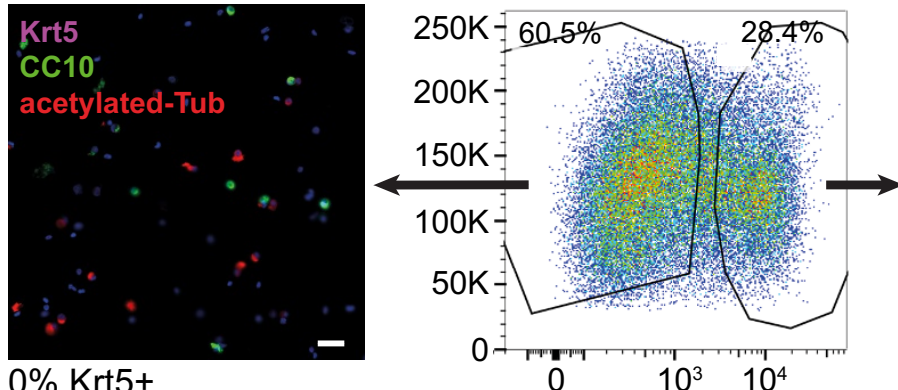

$0 \% \mathrm{Krt5}+$

$\sim 12 \%$ CC $10+$

$\sim 25 \%$ acetylated-Tub+

Integrin $\alpha 6$

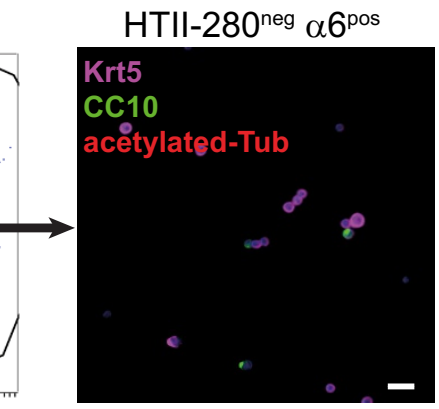

$50-70 \% \mathrm{Krt5}+$

$5-10 \%$ CC $10+$

$0 \%$ acetylated-Tub+

b

Scleroderma Lung Sort

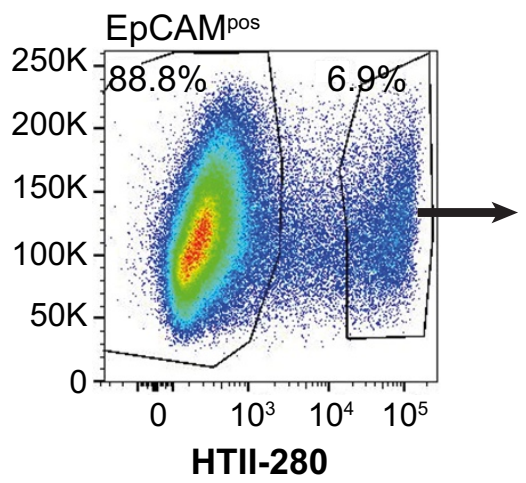

HTII-280 pos

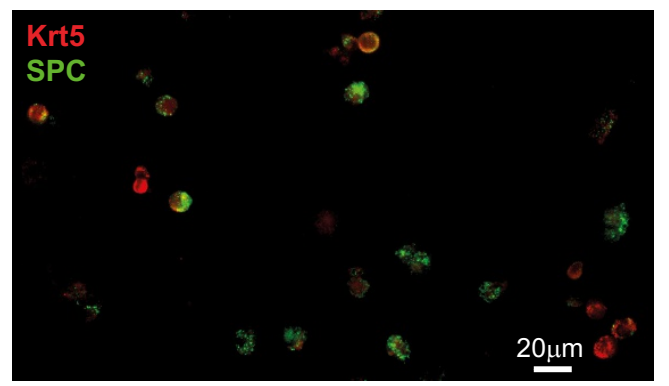

Supplementary Figure 6 Flow plots and cytospin of human lung epithelial cell sort. (a) As judged by cytospin, the HTII-280 ${ }^{\text {os }}$ population captures all the SPC ${ }^{\text {os }}$ AEC2s, and the HTII-280 neg $6^{\text {pos }}$ population is enriched for basal cells in normal human lungs. (b) In scleroderma lung, the percentage of HTII-280 ${ }^{\text {pos }}$ cells as a function of total EpCAMpos cells decreases and both Krt5 ${ }^{\text {pos }}$ and $\mathrm{Krt5} / \mathrm{SPC}$ double positive cells are observed in HTII-280 ${ }^{\text {pos }}$ cells. 
a

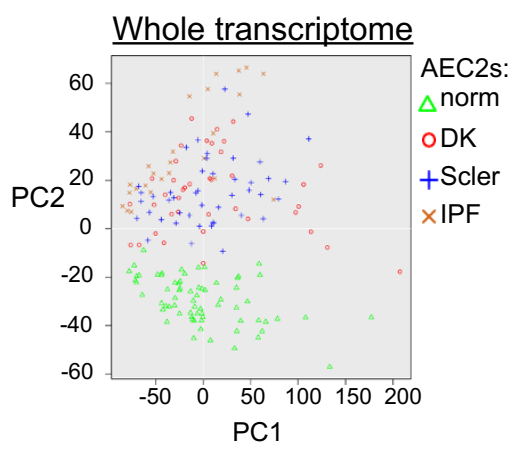

b

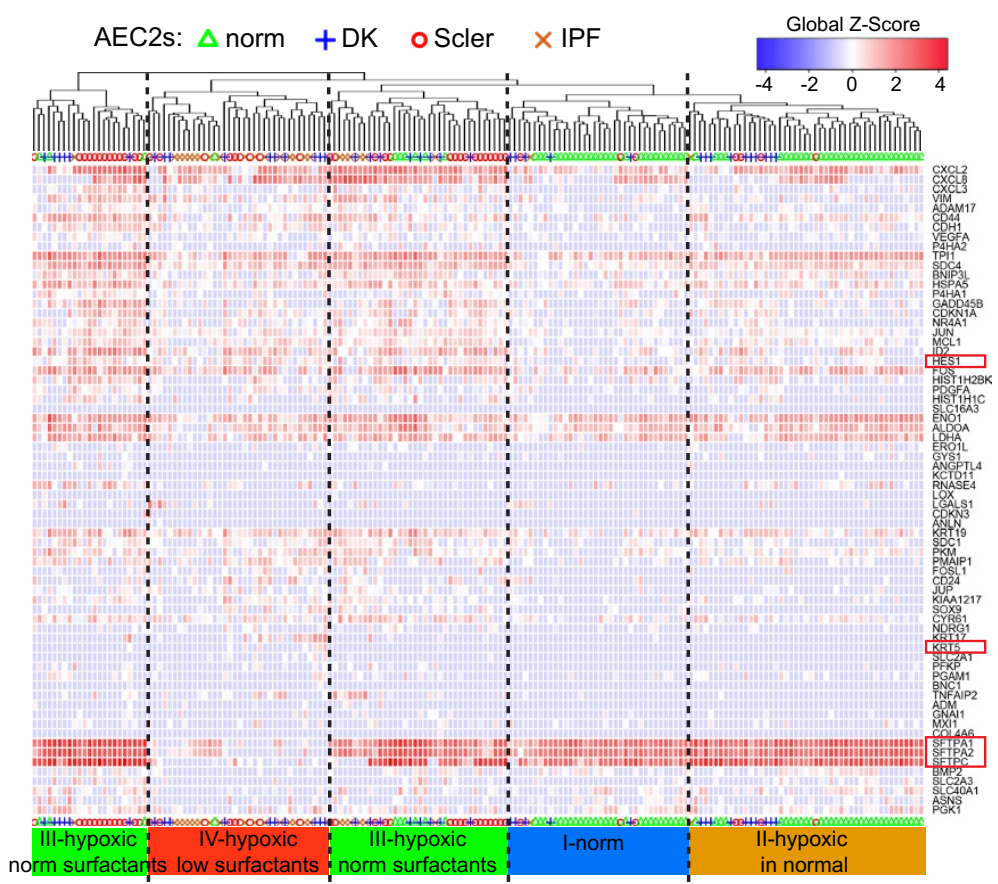

d human AEC2s in vitro

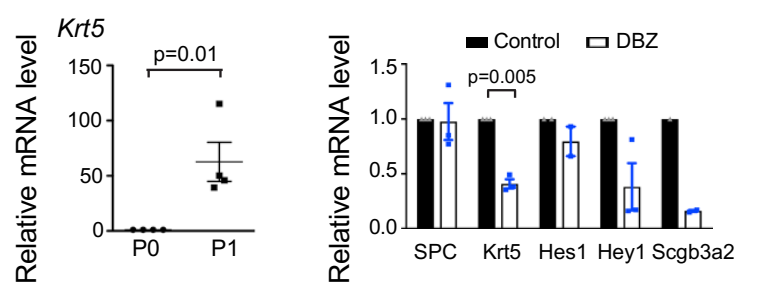

Whole transcriptome minus hypoxia signature and lineage genes

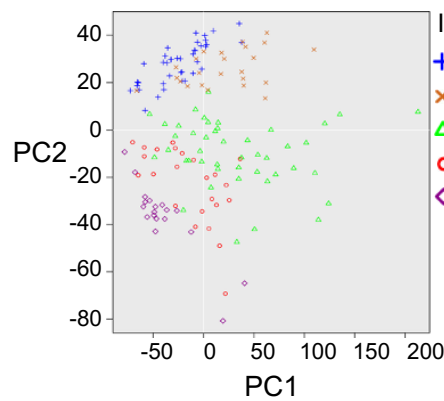

I-IV AEC2s

+ I-norm

$\times$ II-hypoxic in norm

$\triangle$ III-norm SPC

- IV-low SPC

$\diamond \mathrm{V}$-norm basal-enriched

e 102 common genes
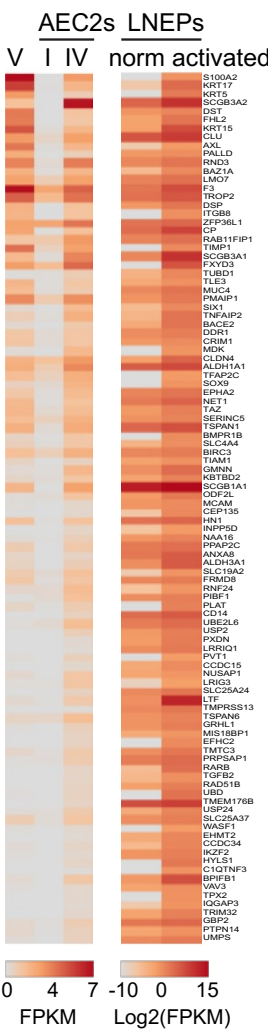

FPKM Log2(FPKM)

Supplementary Figure 7 Transdifferentiation of human AEC2s to basal-like cells and single cell RNA-Seq analysis of primary human lung epithelial cells show distinct hypoxia signature in fibrotic lungs. (a) Whole-genome wide PCA analysis of $\mathrm{HTII}-280^{\text {pos }}$ cells from normal, DK, Scleroderma and IPF lungs, showing AEC2s from fibrotic lungs are distinct from that of normal lungs. (b) Hierarchical clustering of single cell transcriptomes of HTII-280 ${ }^{\text {pos }}$ cells isolated from normal, DK, scleroderma and IPF lungs. Listed genes (rows) are hypoxia signature (listed in Figure 5c) plus STFPA1, STFPA2, SFTPC, KRT5, HES1 (highlighted with red rectangles). Four distinct groups (I-IV) are highlighted. Cells from IPF lungs are mostly in Group IV. (c) PCA analysis of all human cells using the entire genome except for the signature genes from Fig. 6a, demonstrating that the hypoxia \& lineage gene set is predictive of meaningful differences in cell identity at the whole-transcriptome level. (d) Primary human AEC2s (HTII-280 ${ }^{\text {pos}) ~ u p r e g u l a t e d ~ K r t 5 ~ m R N A ~ a f t e r ~ o n e ~}$ passage in culture by qPCR analysis. Notch inhibition (DBZ) during this initial culture attenuated Krt5 upregulation (right). Data are represented as mean $\pm \mathrm{SEM}$, where each point represents one independent cell isolation and experiment ( $n=4$ left, $n=3$ right). $p$ values derived by unpaired two-tailed Student's t test (d, left) or one-sample t-test (d, right). (e) Average FPKM values of human cells (Group I, IV and V), and mouse quiescent and activated LNEPs from RNA-seq are indicated in the heatmap (right) for the shared upregulated 102 gene set (see Fig. 7). 
a

Fig.1d western blot

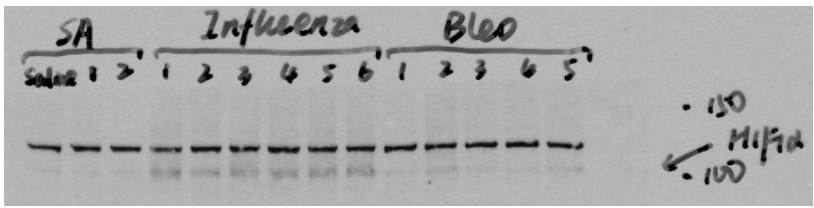

longer exposure of HIF1 $\alpha$ :

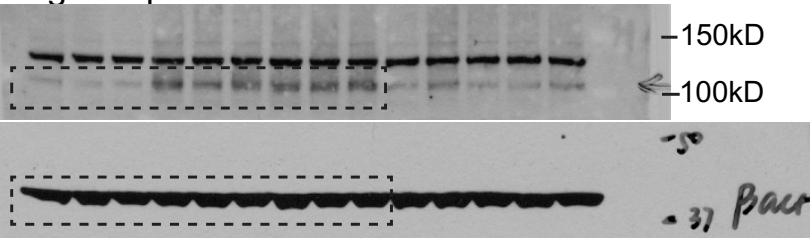

C

Fig.3g DNA gel of CHIP

Krt5 promoter CBE site

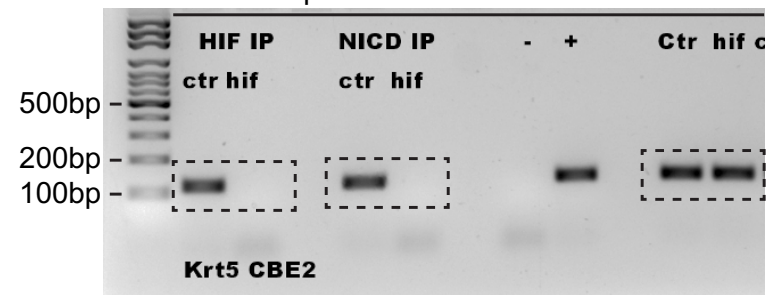

Hey1 promoter CBE site
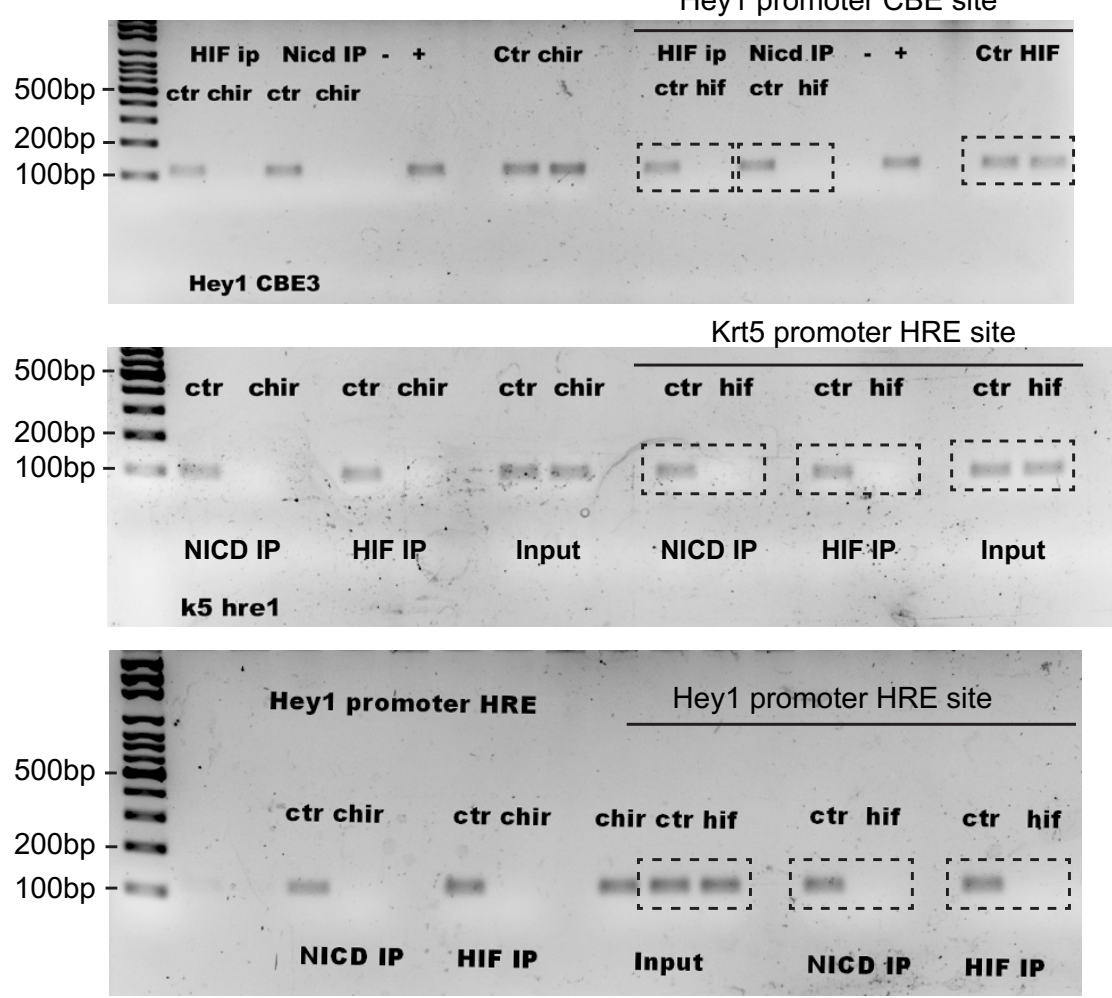

Supplementary Figure 8 Unprocessed original scans of immunoblots and agarose gel electrophoresis images. (a,b,d,f,g,h) Unprocessed western blot b

Fig.3f western blot

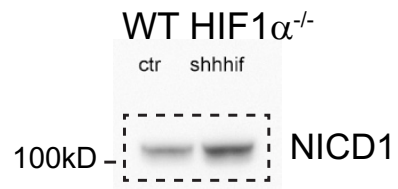

$50 \mathrm{kD}$

37kD-_- 13 -actin
Fig.4f Western blot

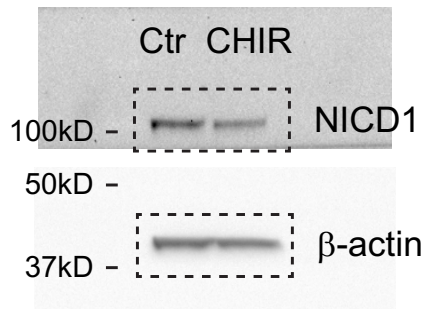


e

Fig.4e DNA gel of CHIP
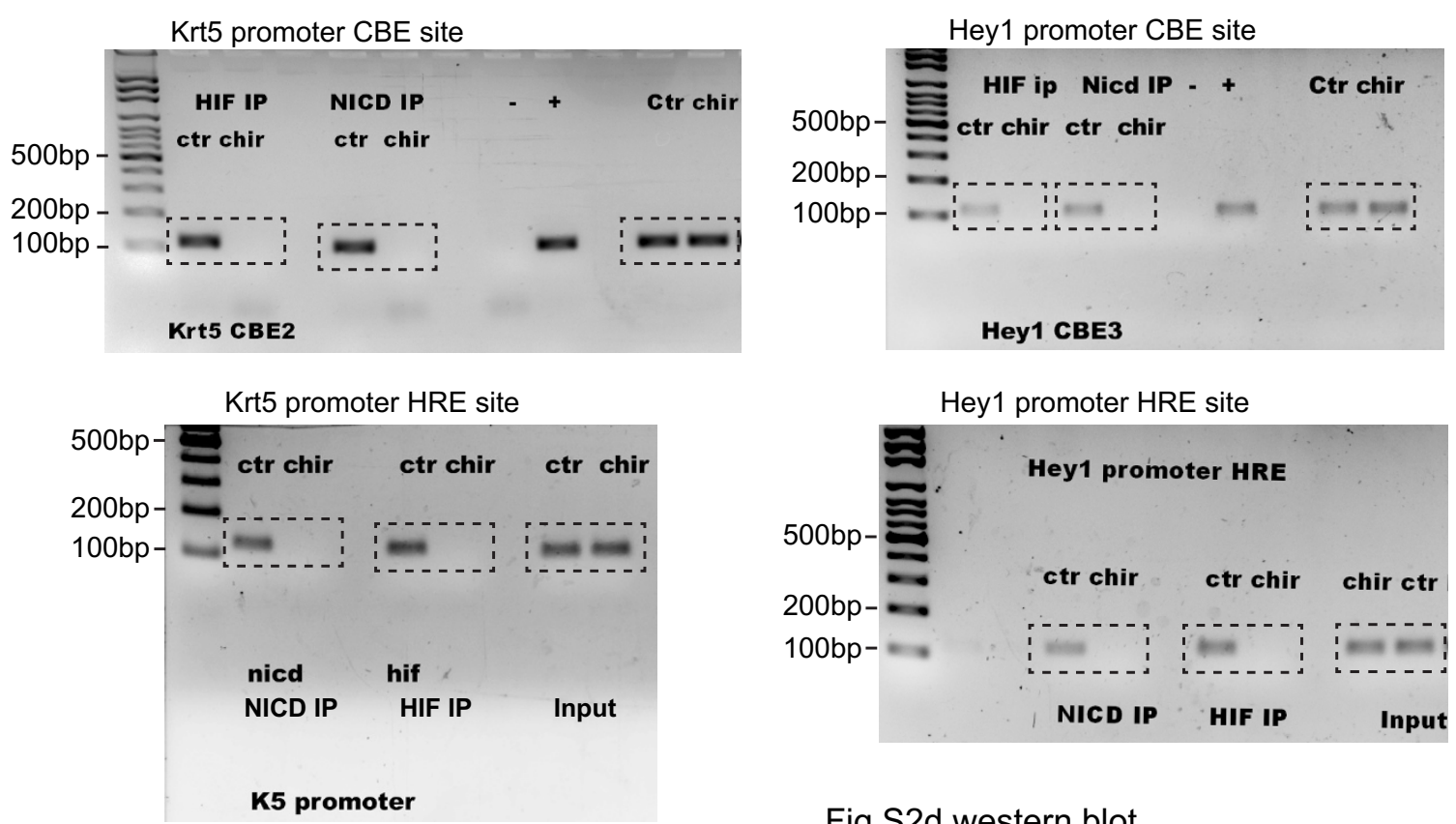

Fig.S2d western blot

$\mathrm{h}$

Fig.5d Western blot
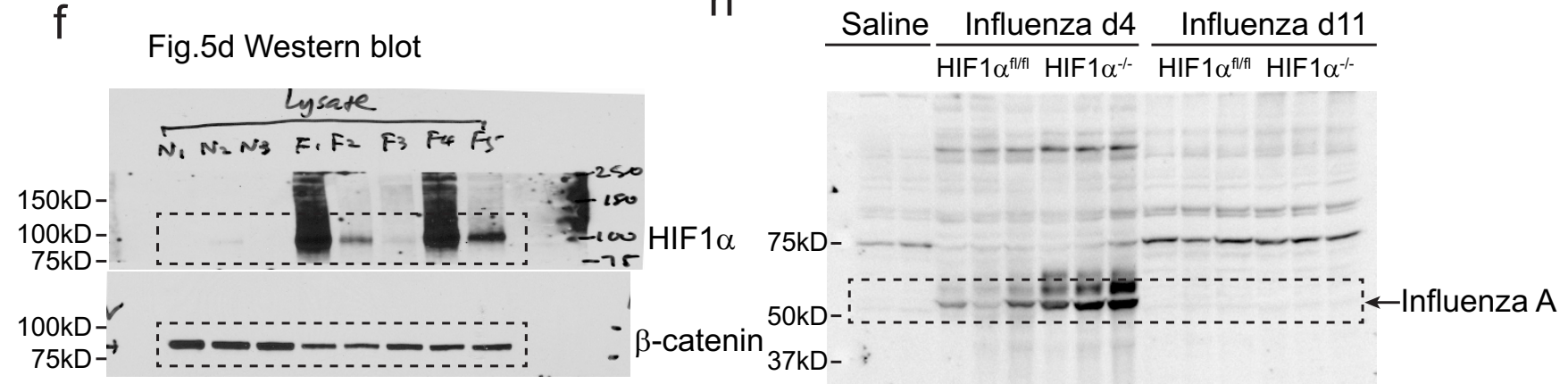

g

Fig.S2a western blot

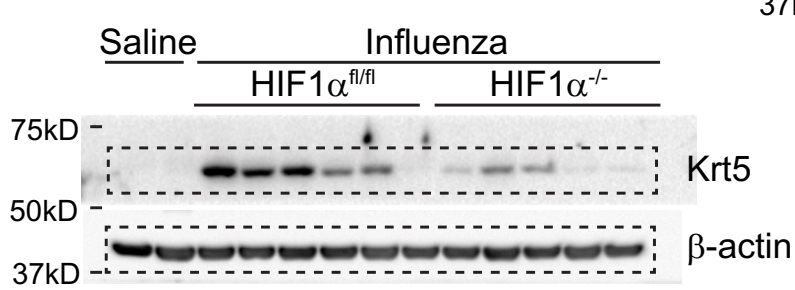

Supplementary Figure 8 Continued 
SUPPLEMENTARY INFORMATION

\section{Supplementary Tables Legends}

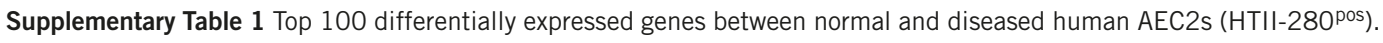

Supplementary Table 2 Top upstream regulators of human diseased versus normal AEC2s (HTII-280 ${ }^{\text {pos }}$ ) from IPA® analysis.

Supplementary Table 3 Expression of hypoxia signature plus HES1, makers of basal cells and AEC2s in normal and diseased human AEC2s (HTII-280pos).

Supplementary Table 4 Top 100 differentially expressed genes in Group I-V cells.

Supplementary Table 5 Expression of top differentially expressed genes and motility genes in Group I, III, IV and V cells.

Supplementary Table 6 Upregulated and downregulated genes shared by activated LNEPs and Group IV hypoxic AEC2s.

Supplementary Table 7 Top upstream regulators of the 102 common genes from IPA® analysis (see excel).

Supplementary Table 8 Pathway analysis of the 102 common genes from IPA® analysis (see excel).

Supplementary Table 9 Diseases and functions affected by the 102 common genes from IPA® analysis (see excel).

Supplementary Table 10 Primers.

Supplementary Table 11 Statistics Source Data. 


\section{Life Sciences Reporting Summary}

Nature Research wishes to improve the reproducibility of the work we publish. This form is published with all life science papers and is intended to promote consistency and transparency in reporting. All life sciences submissions use this form; while some list items might not apply to an individual manuscript, all fields must be completed for clarity.

For further information on the points included in this form, see Reporting Life Sciences Research. For further information on Nature Research policies, including our data availability policy, see Authors \& Referees and the Editorial Policy Checklist.

\section{- Experimental design}

\section{Sample size}

Describe how sample size was determined.

No statistical method was used to predetermine sample size. The sample size was determined on the basis of our prior knowledge of the variability of experimental output and on initial results or pilot experiments for each line of in vitro or in vivo experiments reported.

\section{Data exclusions}

Describe any data exclusions.

No data were excluded from analyses.

\section{Replication}

Describe whether the experimental findings were reliably reproduced. All attempts at replication were successful.

4. Randomization

Describe how samples/organisms/participants were allocated into experimental groups.

The experiment were not randomized.

\section{Blinding}

Describe whether the investigators were blinded to group allocation during data collection and/or analysis.

The investigators were not blinded to group allocation during data collection and/or analysis.

Note: all studies involving animals and/or human research participants must disclose whether blinding and randomization were used.

\section{Statistical parameters}

For all figures and tables that use statistical methods, confirm that the following items are present in relevant figure legends (or the Methods section if additional space is needed).

$\mathrm{n} / \mathrm{a} \mid$ Confirmed

$\square$ The exact sample size $(n)$ for each experimental group/condition, given as a discrete number and unit of measurement (animals, litters, cultures, etc.)

A description of how samples were collected, noting whether measurements were taken from distinct samples or whether the same sample was measured repeatedly.

A statement indicating how many times each experiment was replicated

The statistical test(s) used and whether they are one- or two-sided (note: only common tests should be described solely by name; more complex techniques should be described in the Methods section)

A description of any assumptions or corrections, such as an adjustment for multiple comparisons

The test results (e.g. $p$ values) given as exact values whenever possible and with confidence intervals noted

A summary of the descriptive statistics, including central tendency (e.g. median, mean) and variation (e.g. standard deviation, interquartile range) Clearly defined error bars

See the web collection on statistics for biologists for further resources and guidance. 
Policy information about availability of computer code

\section{Software}

Describe the software used to analyze the data in this study.

Only commercially available or free software was used for data analysis. This includes Graphpad Prism, Fluidigm Singular, FlowJo, and Microsoft Excel.

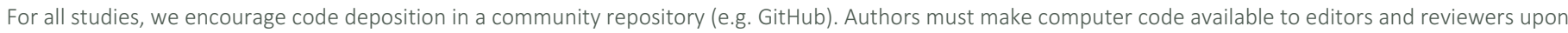
request. The Nature Methods guidance for providing algorithms and software for publication may be useful for any submission.

\section{- Materials and reagents}

Policy information about availability of materials

\section{Materials availability}

Indicate whether there are restrictions on availability of unique materials or if these materials are only available for distribution by a for-profit company.

\section{Antibodies}

Describe the antibodies used and how they were validated for use in the system under study (i.e. assay and species).

\section{Eukaryotic cell lines}

a. State the source of each eukaryotic cell line used.

b. Describe the method of cell line authentication used.

c. Report whether the cell lines were tested for mycoplasma contamination.

d. If any of the cell lines used in the paper are listed in the database of commonly misidentified cell lines maintained by ICLAC, provide a scientific rationale for their use.
Goat anti-CC10 antibody was a gift from Dr. Barry Stripp and HTII-280 antibody was a gift from Dr. Leland Dobbs.

IF: rabbit anti-pro-SPC (1:3000; Millipore, \#AB3786), goat anti-pro-SPC (1:2000; Santa Cruz, \#SC-7706), rabbit anti-Krt5 (1:1000; Covance, \#PRB-160P), chicken anti-Krt5 (1:1000; Covance, \#SIG-3475), rabbit anti$\triangle N p 63$ (1:100; Cell Signaling, \#13109), rat anti-mouse integrin $\beta 4$ (1:200; BD, \#555721), goat anti-CC10 (1:10,000, a gift from Dr. B. Stripp), mouse anti-acetylated tubulin (1:500, Sigma, 6-11B-1), rat anti-E-cadherin (1:500, Invitrogen, \#13-1900), rabbit anti-Hes1 (1:1000; Cell Signaling, \#11988), goat anti-Scgb3a2 (1:100, R\&D, AF3465).

Western blots: HIF1 (1:500, R\&D systems, \#AF1935), pro-SPC (1:500, Millipore, \#AB3786), Krt5 (1:1000, Covance, \#PRB-160P), Cleaved Notch1 (1:1000, Cell signaling, \#4147), E-cadherin (1:2000, BD, \#610181), ?-actin (1:10000, Sigma-Aldrich, \#A5441). To detect influenza A virus, unboiled lysates in $1 \mathrm{mM}$ DTT and 2\% SDS were blotted with anti-Influenza A antibody (1:1000, Millipore, \#AB1074).

FACS: rat anti-mouse CD45 (1:200, BD, \#553078), rat anti-mouse CD16/ CD32 (1:200, BD, \#553143), rat anti-mouse CD31 (1:200, BD, \#553371), then incubated for $1 \mathrm{hr}$ at $4^{\circ} \mathrm{C}$ with the following primary antibodies or viability dye diluted in DMEM (without phenol red) plus 2\% FBS (Gibco): phycoerythrin (PE) or BV421-conjugated rat anti-mouse EpCAM (1:500; Biolegend, \#563477, \#563214), Alexa Fluor ${ }^{\circledR} 647$ or PE-conjugated rat antimouse integrin $\beta 4$ (1:75; BD, \#553745), fixable viability dye eFluor ${ }^{\circledR} 780$ (1:2000, eBioscience), Goat anti-pro-SPC (1:500; Santa Cruz, \#SC-7706), BV421 rat anti-mouse EpCAM, Alexa Fluor ${ }^{\circledR} 488$ donkey anti-goat IgG (1:2000, ThermoFisher, \#A-11055).

Validation for species and application was from manufacturer website.

No eukaryotic cell line was used.

No eukaryotic cell line was used.

No eukaryotic cell line was used.

No commonly misidentified cell lines were used. 


\section{- Animals and human research participants}

Policy information about studies involving animals; when reporting animal research, follow the ARRIVE guidelines

\section{Description of research animals}

Provide details on animals and/or animal-derived materials used in the study.

Policy information about studies involving human research participants

\section{Description of human research participants}

Describe the covariate-relevant population characteristics of the human research participants.
HIF1 $\alpha \mathrm{fl} / \mathrm{fl}$, Shh-Cre, Krt5-CreERT2, Sox2-CreERT2, $\beta$-cateninloxEx3, CC10CreERT, FoxJ1-CreERT2, p63-CreERT2, Ub-GFP, SPC-CreERT2, and Ai14tdTomato mice were used in the study and they were all previously described. For all experiments, $6-8$ week old animals of both sexes were used in equal proportions.

The study did not involved human research participants. All human samples are non-identified, otherwise discarded tissues. 


\section{Flow Cytometry Reporting Summary}

Form fields will expand as needed. Please do not leave fields blank.

\section{- Data presentation}

For all flow cytometry data, confirm that:

$\bigotimes 1$. The axis labels state the marker and fluorochrome used (e.g. CD4-FITC).

$\bigotimes$ 2. The axis scales are clearly visible. Include numbers along axes only for bottom left plot of group (a 'group' is an analysis of identical markers).

\3. All plots are contour plots with outliers or pseudocolor plots.

$\bigotimes 4$. A numerical value for number of cells or percentage (with statistics) is provided.

\section{- Methodological details}

5. Describe the sample preparation.

6. Identify the instrument used for data collection.

7. Describe the software used to collect and analyze the flow cytometry data.

8. Describe the abundance of the relevant cell populations within post-sort fractions.

9. Describe the gating strategy used.
Lung epithelial cells were obtained by Dispase digestion of primary tissue, exactly as described in Methods.

All data was collected on either FACSAria II or LSR II.

Analysis was performed using FlowJo.

Purity checks were routinely performed by re-running 100-500 sorted cells back through the sorter and ensuring they fell within appropriate gates.

As a general rule cells were gated on forward and side scatter corresponding to the known scatter profile of lung epithelial cells. Next they were gated as singlets, and then gated as live (viability dye negative) and CD45 negative. All positive gates were based off of the background fluorescence signal obtained by staining with an isotype antibody conjugated to the same fluorophore as the primary antibody.

Tick this box to confirm that a figure exemplifying the gating strategy is provided in the Supplementary Information. $\bigotimes$ 